\title{
Hopf Bifurcation Analysis of a Synthetic Drug Transmission Model with Time Delays
}

\author{
Zizhen Zhang ${ }^{(D)}$, Fangfang Yang, and Wanjun Xia \\ School of Management Science and Engineering, Anhui University of Finance and Economics, Bengbu 233030, China \\ Correspondence should be addressed to Zizhen Zhang; zzzhaida@163.com
}

Received 16 September 2019; Revised 3 November 2019; Accepted 6 November 2019; Published 22 November 2019

Academic Editor: Toshikazu Kuniya

Copyright (C) 2019 Zizhen Zhang et al. This is an open access article distributed under the Creative Commons Attribution License, which permits unrestricted use, distribution, and reproduction in any medium, provided the original work is properly cited.

\begin{abstract}
This paper is concerned with the Hopf bifurcation of a synthetic drug transmission model with two delays. Firstly, some sufficient conditions of delay-induced bifurcation for such a model are captured by using different combinations of the two delays as the bifurcation parameter. Secondly, based on the center manifold theorem and normal form theory, some sufficient conditions determining properties of the Hopf bifurcation such as the direction and the stability are established. Finally, to underline the effectiveness of the obtained results, some numerical simulations are ultimately addressed.
\end{abstract}

\section{Introduction}

Increased use of heroin and other addictive drugs is an issue of concern all over the world. Drug abuse affects not only life quality of the general public but also the overall situation of social stability and economic development [1-3]. The data from the World Drug Report published by the United Nations (U.N.) showed that thirty-five million people worldwide suffer from drug abuse disorders, and only one-seventh has received treatment [4]. It also showed that, in 2017, millions of people around the world injected drugs, including 1.4 million people living with HIV and 5.6 million people suffering from hepatitis C. From the statistical data, it is clear that the adverse health effect due to drug use is more serious and widespread than previous anticipation, and it is urgent to control the prevalence of addictive drugs.

As stated in [5], the spread of heroin habituation and addiction has similar characteristics to an epidemic, including rapid diffusion and clear geographic boundaries. With the help of epidemic models, one could try to simulate and reveal the nature of epidemics and provide theoretical rules and results for preventing and controlling infectious diseases [6]. In recent decades, mathematical modelling technologies based on the infectious disease models have been developed to understand and combat drug-addiction problems. In [7], White and Comiskey formulated a heroin epidemic model with a standard incidence rate based on principles of mathematical epidemiology. In the succession, Mulone and Straughan [8] found stability conditions for steady states of the proposed model by White and Comiskey. In the subsequent papers, Wang et al. [9-12] studied global stability of a heroin epidemic model with bilinear incidence rate, respectively. Some other works related to the dynamical behaviour of heroin epidemic models with nonlinear incidence rates can be found in $[3,13-15]$, and models with age structure can be found in $[13,15-18]$. For the analytical study of stochastic heroin epidemic models or some other heroin epidemic models, one can also see [1, 2, 19-22].

All the aforementioned models consider only traditional drugs. Compared with traditional drugs, the relevant works are few. On the other hand, synthetic drugs are addictive more easily because they can directly affect the central nervous system as a new type of mental drug. According to China's Drug Situation Report [23], among drug abuse, methamphetamine is the most common. Methamphetamine abusers accounted for $56.1 \%$ of the existing 2.444 million drug addicts, and methamphetamine has replaced heroin as the most abused drug in China. 
Besides, in 2018, the people who relapsed and abused synthetic drugs were accounted for $57.3 \%$ of the total number of drug abuse among the relapsed addicts. Based on the above facts, Ma et al. [24] formulated a synthetic drug transmission model with psychological addicts and general contact rates, and they investigated the local and global stability of the proposed model. However, they assumed that the contact rates of psychological addicts and physiological addicts are equal for the sake of simple calculation and analysis, which is not reasonable because the susceptible individuals who have never taken any drugs are more likely to initiate drug abuse once they contact with the physiological addicts compared to the psychological ones. Based on the work in [24], Saha and Samanta [25] proposed a synthetic drug transmission model with general contact rate and Holling type-II functional responses among susceptible and drug addicts. Recently, Liu et al. [26] proposed the following synthetic drug transmission model with different susceptible compartments:

$$
\left\{\begin{array}{l}
\frac{\mathrm{d} S(t)}{\mathrm{d} t}=\Lambda-\beta_{1} S(t) I(t)-\mu S(t) \\
\frac{\mathrm{d} Q(t)}{\mathrm{d} t}=\varepsilon I(t)+\delta R(t)-\mu Q(t)-\beta_{2} Q(t) I(t) \\
\frac{\mathrm{d} I(t)}{\mathrm{d} t}=\beta_{1} S(t) I(t)+\beta_{2} Q(t) I(t)+\sigma R(t) \\
-(\varepsilon+\gamma+\mu) I(t) \\
\frac{\mathrm{d} R(t)}{\mathrm{d} t}=\gamma I(t)-(\delta+\sigma+\mu) R(t)
\end{array}\right.
$$

where the meanings of $S(t), Q(t), I(t)$, and $R(t)$ are described concisely in Table 1 . All the parameters $\Lambda, \beta_{1}, \mu, \varepsilon, \delta$, $\gamma, \beta_{2}$, and $\sigma$ are positive constants, and their meanings are presented in Table 2. Liu et al. [26] studied the global exponential stability of the drug-free equilibrium and the global stability of the drug-addiction equilibrium, and they showed that special psychological treatment played a very positive role in drug abusers.

It should be pointed out that system (1) assumed that drug abusers can give up drugs instantaneous. This assumption seems not to be realistic since the synthetic drugs are addictive easily and it usually needs a period to give up drugs. Time delays have been incorporated into dynamical models about some other fields by many scholars [27-33]. Generally speaking, delay differential equations exhibit much more complicated dynamics than ordinary differential equations since a time delay could cause the equilibrium of a dynamical model to lose its stability. Hence, it is important to know the critical point at which a synthetic drug transmission model changes its stability. Based on the discussion above, we consider the following synthetic drug transmission model with two time delays:
TABLE 1: The state variables for system (1).

\begin{tabular}{lc}
\hline Parameter & Description \\
\hline$S(t)$ & $\begin{array}{r}\text { Number of susceptible individuals who have never } \\
\text { taken any drugs at time } t\end{array}$ \\
$Q(t)$ & $\begin{array}{c}\text { Number of susceptible individuals who have history } \\
\text { of drug abuse }\end{array}$ \\
$I(t)$ & Number of drug users not in treatment \\
$R(t)$ & Number of individuals who are receiving treatment \\
\hline
\end{tabular}

TABLE 2: The description of parameters for system (1).

\begin{tabular}{lc}
\hline Parameter & Description \\
\hline$\Lambda$ & Immigration rate of the susceptible \\
$\beta_{1}$ & Probability of transmission from $S$ to $I$ \\
$\mu$ & Natural death rate of all populations \\
$\mathrm{E}$ & Self-cure rate from $I$ to $Q$ \\
$\delta$ & Successful treatment rate from $I$ to $Q$ \\
$\gamma$ & Procession rate from $I$ to $R$ \\
$\beta_{2}$ & Probability of transmission from $Q$ to $I$ \\
$\sigma$ & Probability of treatment failure \\
\hline
\end{tabular}$$
\left\{\begin{array}{l}
\frac{\mathrm{d} S(t)}{\mathrm{d} t}=\Lambda-\beta_{1} S(t) I(t)-\mu S(t), \\
\frac{\mathrm{d} Q(t)}{\mathrm{d} t}=\varepsilon I\left(t-\tau_{1}\right)+\delta R\left(t-\tau_{2}\right)-\mu Q(t)-\beta_{2} Q(t) I(t), \\
\frac{\mathrm{d} I(t)}{\mathrm{d} t}=\beta_{1} S(t) I(t)+\beta_{2} Q(t) I(t)+\sigma R(t)-(\gamma+\mu) I(t) \\
-\varepsilon I\left(t-\tau_{1}\right), \\
\frac{\mathrm{d} R(t)}{\mathrm{d} t}=\gamma I(t)-(\sigma+\mu) R(t)-\delta R\left(t-\tau_{2}\right),
\end{array}\right.
$$

where $\tau_{1}$ is the time delay due to the period that the drug abusers use to give up drugs through self-control. $\tau_{2}$ is the time delay due to the period used to give up drugs through successful treatment. The transfer diagram of system (2) is depicted as in Figure 1. This paper mainly concerns the effect of the delays $\tau_{1}$ and $\tau_{2}$ on the stability of system (2).

The framework of the current paper is arranged as follows. In Section 2, the existence of Hopf bifurcation is discussed in detail by using the different combinations of the two delays as the bifurcation parameters and analyzing the distribution of roots of the associated characteristic equations. In Section 3, the direction of Hopf bifurcation and stability of the bifurcating periodic solutions are determined with the help of the center manifold theorem and normal form theory. In Section 4, the effectiveness of the obtained theoretical findings is certified through numerical simulations. Finally, conclusions are drawn in Section 5 . 


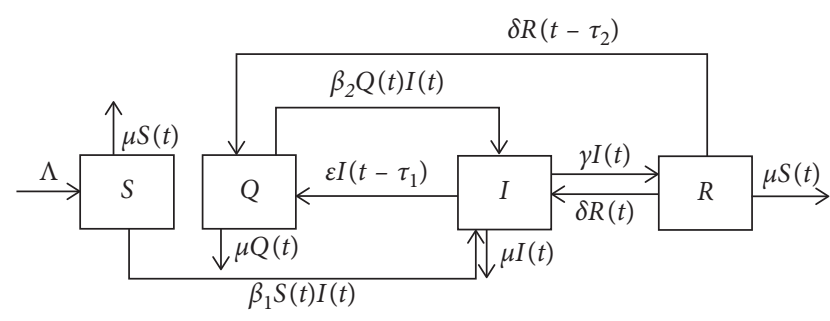

Figure 1: The transfer diagram of system (2).

\section{The Existence of Hopf Bifurcation}

According to the analysis in the literature [26], we know that system (2) has a unique positive synthetic drug-addiction equilibrium $E^{*}\left(S^{*}, Q^{*}, I^{*}, R^{*}\right)$ when

$$
\begin{aligned}
R_{0} & =\frac{\beta_{2} \Lambda(\delta+\sigma+\mu)}{\mu((\varepsilon+\gamma+\mu)(\delta+\sigma+\mu)-\gamma \sigma)}>1, \\
S^{*} & =\frac{\Lambda}{\beta_{1} I^{*}+\mu}, \\
Q^{*} & =\frac{\varepsilon(\delta+\sigma+\mu)+\delta \gamma}{(\delta+\sigma+\mu)\left(\mu+\beta_{2} I^{*}\right)} I^{*}, \\
R^{*} & =\frac{\gamma}{\delta+\sigma+\mu} I^{*},
\end{aligned}
$$

where $I^{*}$ is the positive root of the following equation:

$$
\begin{aligned}
a\left(I^{*}\right)^{2}+b I^{*}+c= & 0, \\
a= & \mu \beta_{1} \beta_{2}(\gamma+\delta+\sigma+\mu), \\
b= & \mu^{2}\left(\beta_{1}+\beta_{2}\right)(\gamma+\delta+\mu+\sigma) \\
& +\mu \varepsilon \beta_{1}(\delta+\sigma+\mu)+\mu \beta_{1} \gamma \delta \\
& -\beta_{1} \beta_{2} \Lambda(\delta+\sigma+\mu), \\
c= & u^{2}(\delta+\sigma+\mu)(\mu+\varepsilon)+\mu^{2} \gamma(\delta+\mu) \\
& -\mu \beta_{1} \Lambda(\delta+\sigma+\mu) .
\end{aligned}
$$

The linear part of system (2) is

$$
\left\{\begin{array}{l}
\frac{\mathrm{d} S(t)}{\mathrm{d} t}=L_{11} S(t)+L_{13} I(t), \\
\frac{\mathrm{d} Q(t)}{\mathrm{d} t}=L_{22} Q(t)+L_{23} I(t)+M_{23} I\left(t-\tau_{1}\right) \\
\quad+N_{24} R\left(t-\tau_{2}\right), \\
\frac{\mathrm{d} I(t)}{\mathrm{d} t}=L_{31} S(t)+L_{32} \mathrm{Q}(t)+L_{33} I(t)+L_{34} R(t) \\
+M_{33} I\left(t-\tau_{1}\right), \\
\frac{\mathrm{d} R(t)}{\mathrm{d} t}=L_{43} I(t)+L_{44} R(t)+N_{44} R\left(t-\tau_{2}\right),
\end{array}\right.
$$

whose characteristic equation is

$$
\begin{aligned}
\lambda^{4} & +a_{3} \lambda^{3}+a_{2} \lambda^{2}+a_{1} \lambda+a_{0}+\left(b_{3} \lambda^{3}+b_{2} \lambda^{2}+b_{1} \lambda+b_{0}\right) e^{-\lambda \tau_{1}} \\
& +\left(c_{3} \lambda^{3}+c_{2} \lambda^{2}+c_{1} \lambda+c_{0}\right) e^{-\lambda \tau_{2}} \\
& +\left(d_{2} \lambda^{2}+d_{1} \lambda+d_{0}\right) e^{-\lambda\left(\tau_{1}+\tau_{2}\right)}=0
\end{aligned}
$$

where

$$
\begin{aligned}
& a_{0}=L_{11} L_{22}\left(L_{33} L_{44}-L_{34} L_{43}\right)+L_{13} L_{31} L_{22} L_{44} \text {, } \\
& a_{1}=\left(L_{34} L_{43}-L_{33} L_{44}\right)\left(L_{11}+L_{22}\right)-L_{13} L_{31}\left(L_{22}+L_{44}\right) \\
& -L_{11} L_{22}\left(L_{33}+L_{44}\right) \\
& a_{2}=\left(L_{11}+L_{22}\right)\left(L_{33}+L_{44}\right)+L_{11} L_{22}+L_{33} L_{44} \\
& -L_{13} L_{31}-L_{34} L_{43} \text {, } \\
& a_{3}=-\left(L_{11}+L_{22}+L_{33}+L_{44}\right) \text {, } \\
& b_{0}=L_{11} L_{44}\left(L_{22} M_{33}-L_{32} M_{23}\right) \text {, } \\
& b_{1}=L_{32} M_{23}\left(L_{11}+L_{44}\right)-M_{33}\left(L_{11} L_{22}+L_{11} L_{44}+L_{22} L_{44}\right) \text {, } \\
& b_{2}=M_{33}\left(L_{11}+L_{22}+L_{44}\right)-L_{32} M_{23} \text {, } \\
& b_{3}=-M_{33} \text {, } \\
& c_{0}=L_{11} L_{22} L_{33} N_{44}+L_{13} L_{31} L_{22} N_{44}+L_{11} L_{32} L_{43} N_{24} \text {, } \\
& c_{1}=-N_{44}\left(L_{11} L_{22}+L_{11} L_{33}+L_{22} L_{33}\right)-L_{13} L_{31} N_{44} \\
& -L_{32} L_{43} N_{24} \text {, } \\
& c_{2}=N_{44}\left(L_{11}+L_{22}+L_{33}\right) \text {, } \\
& c_{3}=-N_{44} \text {, } \\
& d_{0}=L_{11} N_{44}\left(L_{22} M_{33}-L_{32} M_{23}\right) \text {, } \\
& d_{1}=L_{32} M_{23} N_{44}-M_{33} N_{44}\left(L_{11}+L_{22}\right) \text {, } \\
& d_{2}=M_{33} N_{44} \text {, } \\
& L_{11}=-\left(\beta_{1} I^{*}+\mu\right) \text {, } \\
& L_{13}=-\beta_{1} S^{*} \text {, } \\
& L_{22}=-\left(\mu+\beta_{2} I^{*}\right) \text {, } \\
& L_{23}=-\beta_{2} Q^{*} \text {, } \\
& L_{31}=\beta_{1} I^{*} \text {, } \\
& L_{32}=\beta_{2} I^{*} \text {, } \\
& L_{33}=\beta_{1} S^{*}+\beta_{2} Q^{*} \text {, } \\
& L_{34}=\sigma \text {, } \\
& L_{43}=\gamma \text {, } \\
& L_{44}=-(\sigma+\mu) \text {, } \\
& M_{23}=\varepsilon \text {, } \\
& M_{33}=-\varepsilon \text {, } \\
& N_{24}=\delta \text {, } \\
& N_{44}=-\delta \text {. }
\end{aligned}
$$

Case 1. $\tau_{1}=\tau_{2}=0$. When $\tau_{1}=\tau_{2}=0$, equation (6) becomes 


$$
\lambda^{4}+K_{3} \lambda^{3}+K_{2} \lambda^{2}+K_{1} \lambda+K_{0}=0
$$

where

$$
\begin{aligned}
& K_{0}=a_{0}+b_{0}+c_{0}+d_{0}, \\
& K_{1}=a_{1}+b_{1}+c_{1}+d_{1}, \\
& K_{2}=a_{2}+b_{2}+c_{2}+d_{2}, \\
& K_{3}=a_{3}+b_{3}+c_{3} .
\end{aligned}
$$

According to Routh-Hurwitz criterion, the drug-addiction equilibrium $E^{*}\left(S^{*}, Q^{*}, I^{*}, R^{*}\right)$ is locally asymptotically stable provided the following conditions are satisfied: $\left(H_{1}\right) K_{0}>0, K_{3}>0, K_{2} K_{3}>K_{1} K_{4}$, and $K_{1} K_{2} K_{3}>K_{1}^{2} K_{4}+$ $K_{0} K_{3}^{2}$.

Case 2. $\tau_{1}>0$ and $\tau_{2}=0$.

We analyze the effect of $\tau_{1}$ on bifurcation for system (2). When $\tau_{1}>0$ and $\tau_{2}=0$, equation (6) becomes

$$
\begin{aligned}
\lambda^{4} & +K_{13} \lambda^{3}+K_{12} \lambda^{2}+K_{11} \lambda+K_{10} \\
& +\left(L_{13} \lambda^{3}+L_{12} \lambda^{2}+L_{11} \lambda+L_{10}\right) e^{-\lambda \tau_{1}}=0
\end{aligned}
$$

where

$$
\begin{aligned}
& K_{10}=a_{0}+c_{0}, \\
& K_{11}=a_{1}+c_{1}, \\
& K_{12}=a_{2}+c_{2}, \\
& K_{13}=a_{3}+c_{3}, \\
& L_{10}=b_{0}+d_{0}, \\
& L_{11}=b_{1}+d_{1}, \\
& L_{12}=b_{2}+d_{2}, \\
& L_{13}=b_{3} .
\end{aligned}
$$

Assume that $\lambda=i \omega_{1}\left(\omega_{1}>0\right)$ is a purely imaginary root of equation (10), then it follows that

$$
\left\{\begin{array}{l}
\left(L_{10}-L_{12} \omega_{1}^{2}\right) \cos \omega_{1} \tau_{1}-\left(L_{13} \omega_{1}^{3}-L_{11} \omega_{1}\right) \sin \omega_{1} \tau_{1} \\
\quad=K_{12} \omega_{1}^{2}-\omega_{1}^{4}, \\
\left(L_{10}-L_{12} \omega_{1}^{2}\right) \sin \omega_{1} \tau_{1}+\left(L_{13} \omega_{1}^{3}-L_{11} \omega_{1}\right) \cos \omega_{1} \tau_{1} \\
=K_{11} \omega_{1}-K_{13} \omega_{1}^{3} .
\end{array}\right.
$$

By the aid of equation (12), we have

$$
\omega_{1}^{8}+e_{13} \omega_{1}^{6}+e_{12} \omega_{1}^{4}+e_{11} \omega_{1}^{2}+e_{10}=0,
$$

where

$$
\begin{aligned}
& e_{10}=K_{10}^{2}-L_{10}^{2}, \\
& e_{11}=K_{11}^{2}+2 L_{10} L_{12}-2 K_{10} K_{12}-L_{11}^{2}, \\
& e_{12}=K_{12}^{2}+2 K_{10}+2 L_{11} L_{13}-2 K_{11} K_{13}-L_{12}^{2}, \\
& e_{13}=K_{13}^{2}-2 K_{12}-L_{13}^{2} .
\end{aligned}
$$

Making the substitution $v_{1}=\omega_{1}^{2}$, equation (13) can be rewritten as

$$
v_{1}^{4}+e_{13} v_{1}^{3}+e_{12} v_{1}^{2}+e_{11} v_{1}+e_{10}=0
$$

Define

$$
g_{1}\left(v_{1}\right)=v_{1}^{4}+e_{13} v_{1}^{3}+e_{12} v_{1}^{2}+e_{11} v_{1}+e_{10} .
$$

In order to establish the main results of this article, it is assumed that $\left(\mathrm{H}_{21}\right)$ equation (15) has at least one positive real root. Without loss of generality, assume that equation (15) has four positive roots, denoted by $v_{11}, v_{12}, v_{13}$, and $v_{14}$. Accordingly, $\omega_{1 i}=\sqrt{v_{1 i}}(i=1,2,3,4)$ are the roots of equation (13). From equation (12), one has

$$
\tau_{1 i}^{j}=\frac{1}{\omega_{1 i}} \times \arccos \left\{\frac{f_{11}\left(\omega_{1 i}\right)}{f_{12}\left(\omega_{1 i}\right)}+2 n \pi\right\},
$$

with $i=1,2,3,4 ; n=0,1,2, \ldots ;$ and

$$
\begin{aligned}
f_{11}\left(\omega_{1 i}\right)= & \left(L_{12}-K_{13} L_{13}\right) \omega_{1 i}^{6}+\left(K_{11} L_{13}+K_{13} L_{11}\right. \\
& \left.-K_{12} L_{12}-L_{10}\right) \omega_{1 i}^{4}+\left(K_{10} L_{12}+K_{12} L_{10}\right. \\
& \left.-K_{11} L_{11}\right) \omega_{1 i}^{2}-K_{10} L_{10} \\
f_{12}\left(\omega_{1 i}\right)= & L_{13}^{2} \omega_{1 i}^{6}+\left(L_{12}^{2}-2 L_{11} L_{13}\right) \omega_{1 i}^{4} \\
& +\left(L_{11}^{2}-2 L_{10} L_{12}\right) \omega_{1 i}^{2}+L_{10}^{2} .
\end{aligned}
$$

Denote

$$
\begin{array}{r}
\tau_{10}=\tau_{1 i_{0}}^{0}=\min \left\{\tau_{1 i}^{0} \mid i=1,2,3,4\right\}, \\
\omega_{10}=\tau_{1 i_{0}} .
\end{array}
$$

Next, we derive the condition of the occurrence for Hopf bifurcation. Differentiating both sides of equation (10) with regard to $\tau_{1}$ and substituting $\lambda=i \omega_{10}$ into the obtained expression, it can be achieved that

$$
\operatorname{Re}\left[\frac{\mathrm{d} \lambda}{\mathrm{d} \tau_{1}}\right]_{\tau_{1}=\tau_{10}}^{-1}=\frac{g_{1}^{\prime}\left(v_{10}\right)}{\left(L_{13} \omega_{10}^{3}-L_{11} \omega_{10}\right)^{2}+\left(L_{10}-L_{12} \omega_{10}^{2}\right)^{2}}
$$

where $v_{10}=\omega_{10}^{2}$. To ensure the condition of the occurrence for Hopf bifurcation, we educe the following hypothesis: $\left(H_{22}\right) g_{1}^{\prime}\left(v_{10}\right) \neq 0$, which suggests that transversality condition is matched. In conclusion, we have the following results.

Theorem 1. For system (2), if the conditions $\left(H_{1}\right),\left(H_{21}\right)$, and $\left(\mathrm{H}_{22}\right)$ hold, then drug-addiction equilibrium $E^{*}\left(S^{*}, Q^{*}, I^{*}, R^{*}\right)$ is locally asymptotically stable when $\tau_{1} \in\left[0, \tau_{10}\right)$; system (2) undergoes a Hopf bifurcation at the drug-addiction equilibrium $E^{*}\left(S^{*}, Q^{*}, I^{*}, R^{*}\right)$ when $\tau_{1}=\tau_{10}$, and a family of periodic solutions bifurcate from the drugaddiction equilibrium $E^{*}\left(S^{*}, Q^{*}, I^{*}, R^{*}\right)$.

Remark 1. Although the assumptions $\left(\mathrm{H}_{21}\right)$ and $\left(\mathrm{H}_{22}\right)$ seem to be tedious, however, one can verify the assumptions in numerical simulations. 
Case 3. $\tau_{1}=0$ and $\tau_{2}>0$.

Remark 2. When $\tau_{1}=0$ and $\tau_{2}>0$, the analysis of the effect of $\tau_{2}$ on bifurcation for system (2) is similar as that in Case 2. Therefore, we omit it here.

Case 4. $\tau_{1}=\tau_{2}=\tau>0$.

We analyze the effect of $\tau$ on bifurcation for system (2). When $\tau_{1}=\tau_{2}=\tau>0$, equation (6) becomes

$$
\begin{aligned}
\lambda^{4} & +K_{33} \lambda^{3}+K_{32} \lambda^{2}+K_{31} \lambda+K_{30} \\
& +\left(L_{33} \lambda^{3}+L_{32} \lambda^{2}+L_{31} \lambda+L_{30}\right) e^{-\lambda \tau} \\
& +\left(M_{32} \lambda^{2}+M_{31} \lambda+M_{30}\right) e^{-2 \lambda \tau}=0
\end{aligned}
$$

with

$$
\begin{aligned}
K_{30} & =a_{0}, \\
K_{31} & =a_{1}, \\
K_{32} & =a_{2}, \\
K_{33} & =a_{3}, \\
L_{30} & =b_{0}+c_{0}, \\
L_{31} & =b_{1}+c_{1}, \\
L_{32} & =b_{2}+c_{2}, \\
L_{33} & =b_{3}+c_{3}, \\
M_{30} & =d_{0}, \\
M_{31} & =d_{1}, \\
M_{32} & =d_{2} .
\end{aligned}
$$

By multiplying $e^{\lambda \tau}$ on both sides of equation (21), it can be procured that

$$
\begin{aligned}
& L_{33} \lambda^{3}+L_{32} \lambda^{2}+L_{31} \lambda+L_{30} \\
& +\left(\lambda^{4}+K_{33} \lambda^{3}+K_{32} \lambda^{2}+K_{31} \lambda+K_{30}\right) e^{\lambda \tau} \\
& \quad+\left(M_{32} \lambda^{2}+M_{31} \lambda+M_{30}\right) e^{-\lambda \tau}=0 .
\end{aligned}
$$

Suppose $i \omega(\omega>0)$ is the root of equation (23), then one has

$$
\left\{\begin{array}{l}
J_{41}(\omega) \cos \omega \tau+J_{42}(\omega) \sin \omega \tau=J_{45}(\omega), \\
J_{43}(\omega) \sin \omega \tau-J_{44}(\omega) \cos \omega \tau=J_{46}(\omega),
\end{array}\right.
$$

where

$$
\begin{aligned}
& J_{41}(\omega)=\omega^{4}-\left(K_{32}+M_{32}\right) \omega^{2}+K_{30}+M_{30}, \\
& J_{42}(\omega)=K_{33} \omega^{3}-\left(K_{31}-M_{31}\right) \omega, \\
& J_{43}(\omega)=\omega^{4}-\left(K_{32}-M_{32}\right) \omega^{2}+K_{30}-M_{30}, \\
& J_{44}(\omega)=K_{33} \omega^{3}-\left(K_{31}+M_{31}\right) \omega, \\
& J_{45}(\omega)=L_{32} \omega^{2}-L_{30}, \\
& J_{46}(\omega)=L_{33} \omega^{3}-L_{31} \omega .
\end{aligned}
$$
obtains

$$
\begin{aligned}
& \cos \omega \tau=\frac{l_{6} \omega^{6}+l_{4} \omega^{4}+l_{2} \omega^{2}+l_{0}}{\omega^{8}+j_{6} \omega^{6}+j_{4} \omega^{4}+j_{2} \omega^{2}+j_{0}}, \\
& \sin \omega \tau=\frac{l_{7} \omega^{7}+l_{5} \omega^{5}+l_{3} \omega^{3}+l_{1} \omega}{\omega^{8}+j_{6} \omega^{6}+j_{4} \omega^{4}+j_{2} \omega^{2}+j_{0}},
\end{aligned}
$$

with

$$
\begin{aligned}
j_{0}= & K_{30}^{2}-M_{30}^{2}, \\
j_{2}= & K_{31}^{2}-M_{31}^{2}-2 K_{30} K_{32}+2 M_{30} M_{32}, \\
j_{4}= & 2 K_{30}-M_{32}^{2}-2 K_{31} K_{33}, \\
j_{6}= & K_{33}^{2}-2 K_{32}, \\
l_{0}= & -\left(K_{30}-M_{30}\right) L_{30}, \\
l_{1}= & \left(K_{31}+M_{31}\right) L_{30}-\left(K_{30}+M_{30}\right) L_{31}, \\
l_{2}= & \left(K_{32}-M_{32}\right) L_{30}+\left(K_{30}-M_{30}\right) L_{32}-\left(K_{31}-M_{31}\right) L_{31}, \\
l_{3}= & \left(K_{32}+M_{32}\right) L_{31}+\left(K_{30}+M_{30}\right) L_{33}-\left(K_{31}+M_{31}\right) L_{32} \\
& -K_{33} L_{30}, \\
l_{4}= & \left(K_{31}-M_{31}\right) L_{33}-\left(K_{32}-M_{32}\right) L_{32}+K_{33} L_{31}-L_{30}, \\
l_{5}= & K_{33} L_{32}-L_{31}-\left(K_{32}-M_{32}\right) L_{33}, \\
l_{6}= & L_{32}-K_{33} L_{33}, \\
l_{7}= & L_{33} .
\end{aligned}
$$

Squaring both sides of equations (26) and (27), respectively, and adding them together, one has

$$
\begin{aligned}
& \omega^{16}+k_{7} \omega^{14}+k_{6} \omega^{12}+k_{5} \omega^{10}+k_{4} \omega^{8}+k_{3} \omega^{6}+k_{2} \omega^{4} \\
& +k_{1} \omega^{2}+k_{0}=0,
\end{aligned}
$$

where

$$
\begin{aligned}
& k_{0}=j_{0}^{2}-l_{0}^{2}, \\
& k_{1}=2 j_{0} j_{2}-2 l_{0} l_{2}-l_{1}^{2}, \\
& k_{2}=j_{2}^{2}+2 j_{0} j_{4}-l_{2}^{2}-2 l_{0} l_{4}-2 l_{1} l_{3}, \\
& k_{3}=2 j_{0} j_{6}+2 j_{2} j_{4}-2 l_{0} l_{6}-2 l_{2} l_{4}-l_{3}^{2}-2 l_{1} l_{5}, \\
& k_{4}=j_{4}^{2}+2 j_{0}+2 j_{2} j_{6}-l_{4}^{2}-2 l_{2} l_{6}-2 l_{1} l_{7}-2 l_{3} l_{5}, \\
& k_{5}=2 j_{2}+2 j_{4} j_{6}-2 l_{4} l_{6}-l_{5}^{2}-2 l_{3} l_{7}, \\
& k_{6}=j_{6}^{2}+2 j_{4}-l_{6}^{2}-2 l_{5} l_{7}, k_{7}=2 j_{6}-l_{7}^{2} .
\end{aligned}
$$

Let $\omega^{2}=v$, then equation (29) becomes

$v^{8}+k_{7} v^{7}+k_{6} v^{6}+k_{5} v^{5}+k_{4} v^{4}+k_{3} v^{3}+k_{2} v^{2}+k_{1} v+k_{0}=0$.

Suppose that $\left(H_{41}\right)$ equation (29) has at least one positive root. Without loss of generality, assume that equation (31) has eight positive roots, denoted by 
$v_{1}, v_{2}, \ldots, v_{8}$. Accordingly, $\omega_{i}=\sqrt{v_{i}}(i=1,2, \ldots, 8)$ are the roots of equation (29). Thus, one has

$$
\tau_{i}^{j}=\frac{1}{\omega_{i}} \times \arccos \left\{\frac{l_{6} \omega_{i}^{6}+l_{4} \omega_{i}^{4}+l_{2} \omega_{i}^{2}+l_{0}}{\omega_{i}^{8}+j_{6} \omega_{i}^{6}+j_{4} \omega_{i}^{4}+j_{2} \omega_{i}^{2}+j_{0}}+2 n \pi\right\},
$$

with $i=1,2, \ldots, 8 ; n=0,1,2, \ldots ;$ and

$$
\begin{array}{r}
\tau_{0}=\tau_{i_{0}}^{0}=\min \left\{\tau_{i}^{0} \mid i=1,2, \ldots, 8\right\}, \\
\omega_{0}=\tau_{i_{0}} .
\end{array}
$$

Differentiating both sides of equation (23) with respect to $\tau$ yields

$$
\left[\frac{\mathrm{d} \lambda}{\mathrm{d} \tau}\right]^{-1}=-\frac{f_{31}(\lambda)}{f_{32}(\lambda)}-\frac{\tau}{\lambda}
$$

where

$$
\begin{aligned}
f_{31}(\lambda)= & 3 L_{33} \lambda^{2}+2 L_{32} \lambda+L_{31}+\left(4 \lambda^{3}+3 K_{33} \lambda^{2}+2 K_{32} \lambda\right. \\
& \left.+K_{31}\right) e^{\lambda \tau}+\left(2 M_{32} \lambda+M_{31}\right) e^{-\lambda \tau} \\
f_{32}(\lambda)= & \lambda\left(\lambda^{4}+K_{33} \lambda^{3}+K_{32} \lambda^{2}+K_{31} \lambda+K_{30}\right) e^{\lambda \tau} \\
& -\lambda\left(M_{32} \lambda^{2}+M_{31} \lambda+M_{30}\right) e^{-\lambda \tau} .
\end{aligned}
$$

Hence, one obtains

$$
\operatorname{Re}\left[\frac{\mathrm{d} \lambda}{\mathrm{d} \tau}\right]_{\tau=\tau_{0}}^{-1}=\frac{U_{3 R} V_{3 R}+U_{3 I} V_{3 I}}{V_{3 R}^{2}+V_{3 I}^{2}}
$$

with

$$
\begin{aligned}
U_{3 R}= & L_{31}-3 L_{33} \omega_{0}^{2}+\left(K_{31}+M_{31}-3 K_{33} \omega_{0}^{2}\right) \cos \omega_{0} \tau_{0} \\
& -\left(2 K_{32} \omega_{0}-2 M_{32} \omega_{0}-4 \omega_{0}^{3}\right) \sin \omega_{0} \tau_{0}, \\
U_{3 I}= & 2 L_{32} \omega_{0}+\left(K_{31}-M_{31}-3 K_{33} \omega_{0}^{2}\right) \sin \omega_{0} \tau_{0} \\
& +\left(2 K_{32} \omega_{0}+2 M_{32} \omega_{0}-4 \omega_{0}^{3}\right) \cos \omega_{0} \tau_{0}, \\
V_{3 R}= & \left(K_{33} \omega_{0}^{4}-K_{31} \omega_{0}^{2}+M_{31} \omega_{0}^{2}\right) \cos \omega_{0} \tau_{0} \\
& -\left(\omega_{0}^{5}-K_{32} \omega_{0}^{3}-M_{32} \omega_{0}^{3}+K_{30} \omega_{0}+M_{30} \omega_{0}\right) \sin \omega_{0} \tau_{0}, \\
V_{3 I}= & \left(K_{33} \omega_{0}^{4}-K_{31} \omega_{0}^{2}-M_{31} \omega_{0}^{2}\right) \sin \omega_{0} \tau_{0} \\
& +\left(\omega_{0}^{5}-K_{32} \omega_{0}^{3}+M_{32} \omega_{0}^{3}+K_{30} \omega_{0}-M_{30} \omega_{0}\right) \cos \omega_{0} \tau_{0} .
\end{aligned}
$$

In order to obtain the main results, it is necessary to make the following extra assumption $\left(H_{42}\right) U_{3 R} V_{3 R}+$ $U_{3 I} V_{3 I} \neq 0$, which ensures that transversality condition holds.

Theorem 2. For system (2), if the conditions $\left(H_{1}\right),\left(H_{41}\right)$, and $\left(\mathrm{H}_{42}\right)$ hold, then drug-addiction equilibrium $E^{*}\left(S^{*}, Q^{*}, I^{*}, R^{*}\right)$ is locally asymptotically stable when $\tau \in\left[0, \tau_{0}\right)$; system (2) undergoes a Hopf bifurcation at the drug-addiction equilibrium $E^{*}\left(S^{*}, Q^{*}, I^{*}, R^{*}\right)$ when $\tau=\tau_{0}$, and a family of periodic solutions bifurcate from the drugaddiction equilibrium $E^{*}\left(S^{*}, Q^{*}, I^{*}, R^{*}\right)$.

Remark 3. Although the assumptions $\left(H_{41}\right)$ and $\left(H_{42}\right)$ seem to be tedious, however, one can verify the assumptions in numerical simulations.

Case 5. $\tau_{1} \in\left(0, \tau_{10}\right)$ and $\tau_{2}>0$.

Motivated by the work in [34], in this case, we analyze the effect of $\tau_{2}$ on bifurcation for system (2) and fix $\tau_{1} \in\left(0, \tau_{10}\right)$. Suppose $i \omega_{2}^{\prime}\left(\omega_{2}^{\prime}>0\right)$ is the root of equation (6). For convenience, we still denote $\omega_{2}^{\prime}$ as $\omega_{2}$. Then, one has

$$
\left\{\begin{array}{l}
J_{41}\left(\omega_{2}\right) \sin \omega_{2} \tau_{2}+J_{42}\left(\omega_{2}\right) \cos \omega_{2} \tau_{2}=J_{43}\left(\omega_{2}\right), \\
J_{41}\left(\omega_{2}\right) \cos \omega_{2} \tau_{2}-J_{42}\left(\omega_{2}\right) \sin \omega_{2} \tau_{2}=J_{44}\left(\omega_{2}\right),
\end{array}\right.
$$

where

$$
\begin{aligned}
J_{41}\left(\omega_{2}\right)= & c_{1} \omega_{2}-c_{3} \omega_{2}^{3}+d_{1} \omega_{2} \cos \omega_{2} \tau_{1} \\
& -\left(d_{0}-d_{2} \omega_{2}^{2}\right) \sin \omega_{2} \tau_{1}, \\
J_{42}\left(\omega_{2}\right)= & c_{0}-c_{2} \omega_{2}^{2}+d_{1} \omega_{2} \sin \omega_{2} \tau_{1}+\left(d_{0}-d_{2} \omega_{2}^{2}\right) \cos \omega_{2} \tau_{1}, \\
J_{43}\left(\omega_{2}\right)= & a_{2} \omega_{2}^{2}-\omega_{2}^{4}-a_{0}-\left(b_{1} \omega_{2}-b_{3} \omega_{2}^{3}\right) \sin \omega_{2} \tau_{1} \\
& -\left(b_{0}-b_{2} \omega_{2}^{2}\right) \cos \omega_{2} \tau_{1}, \\
J_{44}\left(\omega_{2}\right)= & a_{3} \omega_{2}^{3}-a_{1} \omega_{2}-\left(b_{1} \omega_{2}-b_{3} \omega_{2}^{3}\right) \cos \omega_{2} \tau_{1} \\
& +\left(b_{0}-b_{2} \omega_{2}^{2}\right) \sin \omega_{2} \tau_{1} .
\end{aligned}
$$

Squaring both sides of the above equations, respectively, and adding them together, one has

$$
r_{40}\left(\omega_{2}\right)+2 r_{41}\left(\omega_{2}\right) \cos \omega_{2} \tau_{1}+2 r_{42}\left(\omega_{2}\right) \sin \omega_{2} \tau_{1}=0,
$$

where

$$
\begin{aligned}
r_{40}\left(\omega_{2}\right)= & \omega_{2}^{8}+\left(a_{3}^{2}-2 a_{2}-b_{3}^{2}-c_{3}^{2}\right) \omega_{2}^{6} \\
& +\left(a_{2}^{2}+2 a_{0}-2 a_{1} a_{3}+b_{2}^{2}-2 b_{1} b_{3}\right. \\
& \left.-c_{2}^{2}+2 c_{1} c_{3}-d_{2}^{2}\right) \omega_{2}^{4}+\left(a_{1}^{2}-2 a_{0} a_{2}-2 b_{0} b_{2}+b_{1}^{2}\right. \\
& \left.-c_{1}^{2}+2 c_{0} c_{2}-d_{1}^{2}+2 d_{0} d_{2}\right) \omega_{2}^{2}+a_{0}^{2}+b_{0}^{2}-c_{0}^{2}-d_{0}^{2}, \\
r_{41}\left(\omega_{2}\right)= & \left(a_{3} b_{3}-b_{2}\right) \omega_{2}^{6}+\left(a_{2} b_{2}-a_{1} b_{3}-a_{3} b_{1}+b_{0}\right. \\
& \left.-c_{2} d_{2}+c_{3} d_{1}\right) \omega_{2}^{4}+\left(a_{1} b_{1}-a_{2} b_{0}-a_{0} b_{2}+c_{0} d_{2}\right. \\
& \left.-c_{1} d_{1}+c_{2} d_{0}\right) \omega_{2}+a_{0} b_{0}-c_{0} d_{0}, \\
r_{42}\left(\omega_{2}\right)= & -b_{3} \omega_{2}^{7}+\left(a_{2} b_{3}+b_{1}-a_{3} b_{2}+c_{3} d_{2}\right) \omega_{2}^{5} \\
& +\left(a_{3} b_{0}+a_{1} b_{2}-a_{2} b_{1}-a_{0} b_{3}-c_{1} d_{2}\right. \\
& \left.+c_{2} d_{1}-c_{3} d_{0}\right) \omega_{2}^{3}+\left(a_{0} b_{1}-a_{1} b_{0}-c_{0} d_{1}+c_{1} d_{0}\right) \omega_{2} .
\end{aligned}
$$

Suppose that $\left(H_{51}\right)$ equation (40) has finite positive roots, denoted by $\omega_{21 *}, \omega_{22 *}, \ldots, \omega_{2 i *}$. Thus, 


$$
\tau_{2 i *}^{j}=\frac{1}{\omega_{2 i *}} \times \arccos \left\{\frac{J_{41}\left(\omega_{2 i *}\right) \times J_{44}\left(\omega_{2 i *}\right)+J_{42}\left(\omega_{2 i *}\right) \times J_{43}\left(\omega_{2 i *}\right)}{J_{41}^{2}\left(\omega_{2 i *}\right)+J_{42}^{2}\left(\omega_{2 i *}\right)}+2 n \pi\right\},
$$

with $i=1,2, \ldots, k ; n=0,1,2, \ldots$; and

$$
\tau_{2 *}=\tau_{2 i *_{0}}^{0}=\min \left\{\tau_{2 i *}^{0} \mid i=1,2, \ldots, k\right\} .
$$

$$
\left[\frac{\mathrm{d} \lambda}{\mathrm{d} \tau}\right]^{-1}=-\frac{f_{41}(\lambda)}{f_{42}(\lambda)}-\frac{\tau_{2}}{\lambda}
$$

Differentiating equation (6) with respect to $\tau_{2}$, one has

$$
\begin{aligned}
f_{41}(\lambda)= & 4 \lambda^{3}+3 a_{3} \lambda^{2}+2 a_{2} \lambda+a_{1}+\left(\left(3 b_{3}-\tau_{1} b_{2}\right) \lambda^{2}-\tau_{1} \lambda^{3}+\left(2 b_{2}-\tau_{1} b_{1}\right) \lambda+b_{1}-\tau_{1} b_{0}\right) e^{-\lambda \tau_{1}} \\
& +\left(\left(2 d_{2}-\tau_{1} d_{1}\right) \lambda-\tau_{1} d_{2} \lambda^{2}+d_{1}-\tau_{1} d_{0}\right) e^{-\lambda\left(\tau_{1}+\tau_{2}\right)}+\left(3 c_{3} \lambda^{2}+2 c_{2} \lambda+c_{1}\right) e^{-\lambda \tau_{2}} \\
f_{42}(\lambda)= & \lambda\left(c_{3} \lambda^{3}+c_{2} \lambda^{2}+c_{1} \lambda+c_{0}\right) e^{-\lambda \tau_{2}}+\lambda\left(d_{2} \lambda^{2}+d_{1} \lambda+d_{0}\right) e^{-\lambda\left(\tau_{1}+\tau_{2}\right)} .
\end{aligned}
$$

Thus, one obtains

with

$$
\operatorname{Re}\left[\frac{\mathrm{d} \lambda}{\mathrm{d} \tau_{2}}\right]_{\tau_{2}=\tau_{2 *}}^{-1}=\frac{U_{4 R} V_{4 R}+U_{4 I} V_{4 I}}{V_{4 R}^{2}+V_{4 I}^{2}}
$$

$$
\begin{aligned}
U_{4 R}= & 2 c_{2} \omega_{2 *} \sin \omega_{2 *} \tau_{2 *}+\left(c_{1}-3 c_{3} \omega_{2 *}^{2}\right) \cos \omega_{2 *} \tau_{2 *}+a_{1}-3 a_{3} \omega_{2 *}^{2}+\left(2 d_{2}-\tau_{1} d_{1}\right) \omega_{2 *}\left(\sin \omega_{2 *} \tau_{1} \cos \omega_{2 *} \tau_{2 *}\right. \\
& \left.+\cos \omega_{2 *} \tau_{1} \sin \omega_{2 *} \tau_{2 *}\right)+\left(\tau_{1} d_{2} \omega_{2 *}^{2}+d_{1}-\tau_{1} d_{0}\right)\left(\cos \omega_{2 *} \tau_{1} \cos \omega_{2 *} \tau_{2 *}-\sin \omega_{2 *} \tau_{1} \sin \omega_{2 *} \tau_{2 *}\right) \\
& +\left(\tau_{1} \omega_{2 *}^{3}+\left(2 b_{2}-\tau_{1} b_{1}\right) \omega_{2 *}\right) \sin \omega_{2 *} \tau_{1}+\left(\left(\tau_{1} b_{2}-3 b_{3}\right) \omega_{2 *}^{2}+b_{1}-\tau_{1} b_{0}\right) \cos \omega_{2 *} \tau_{1} \\
U_{4 I}= & 2 c_{2} \omega_{2 *} \cos \omega_{2 *} \tau_{2 *}-\left(c_{1}-3 c_{3} \omega_{2 *}^{2}\right) \sin \omega_{2 *} \tau_{2 *}+2 a_{2} \omega_{2 *}-4 \omega_{2 *}^{3}+\left(2 d_{2}-\tau_{1} d_{1}\right) \omega_{2 *}\left(\cos \omega_{2 *} \tau_{1} \cos \omega_{2 *} \tau_{2 *}\right. \\
& \left.-\sin \omega_{2 *} \tau_{1} \sin \omega_{2 *} \tau_{2 *}\right)-\left(\tau_{1} d_{2} \omega_{2 *}^{2}+d_{1}-\tau_{1} d_{0}\right)\left(\sin \omega_{2 *} \tau_{1} \cos \omega_{2 *} \tau_{2 *}+\cos \omega_{2 *} \tau_{1} \sin \omega_{2 *} \tau_{2 *}\right) \\
& +\left(\tau_{1} \omega_{2 *}^{3}+\left(2 b_{2}-\tau_{1} b_{1}\right) \omega_{2 *}\right) \cos \omega_{2 *} \tau_{1}-\left(\left(\tau_{1} b_{2}-3 b_{3}\right) \omega_{2 *}^{2}+b_{1}-\tau_{1} b_{0}\right) \sin \omega_{2 *} \tau_{1} \\
V_{4 R}= & \left(d_{0} \omega_{2 *}-d_{2} \omega_{2 *}^{3}\right)\left(\sin \omega_{2 *} \tau_{1} \cos \omega_{2 *} \cos \omega_{2 *}+\cos \omega_{2 *} \tau_{1} \sin \omega_{2 *} \tau_{2 *}\right)-d_{1} \omega_{2 *}^{2}\left(\cos \omega_{2 *} \tau_{1} \cos \omega_{2 *} \tau_{2 *}\right. \\
& \left.-\sin \omega_{2 *} \tau_{1} \sin \omega_{2 *} \tau_{2 *}\right) \\
V_{4 I}= & \left(d_{0} \omega_{2 *}-d_{2} \omega_{2 *}^{3}\right)\left(\cos \omega_{2 *} \tau_{1} \cos \omega_{2 *} \cos \omega_{2 *}-\sin \omega_{2 *} \tau_{1} \sin \omega_{2 *} \tau_{2 *}\right)+d_{1} \omega_{2 *}^{2}\left(\sin \omega_{2 *} \tau_{1} \cos \omega_{2 *} \tau_{2 *}\right. \\
& \left.+\cos \omega_{2 *} \tau_{1} \sin \omega_{2 *} \tau_{2 *}\right) .
\end{aligned}
$$

As in Case 4, we make the following extra assumption $\left(H_{52}\right) U_{34} V_{4 R}+U_{4 I} V_{4 I} \neq 0$, which ensures that transversality condition holds.

Theorem 3. For system (2), if $\tau_{1} \in\left(0, \tau_{10}\right)$ and $\tau_{2}>0$ and the conditions $\left(H_{1}\right),\left(H_{51}\right)$, and $\left(H_{52}\right)$ hold, then drug-addiction equilibrium $E^{*}\left(S^{*}, Q^{*}, I^{*}, R^{*}\right)$ is locally asymptotically stable when $\tau_{2} \in\left[0, \tau_{2 *}\right)$; system (2) undergoes a Hopf bifurcation at the drug-addiction equilibrium $E^{*}\left(S^{*}, Q^{*}, I^{*}, R^{*}\right)$ when $\tau_{2}=\tau_{2 *}$, and a family of periodic solutions bifurcate from the drug-addiction equilibrium $E^{*}\left(S^{*}, Q^{*}, I^{*}, R^{*}\right)$.
Remark 4. Although the assumptions $\left(H_{51}\right)$ and $\left(H_{52}\right)$ seem to be tedious, however, one can verify the assumptions in numerical simulations.

\section{Properties of Hopf Bifurcation}

In this section, by employing the center manifold theorem and normal form theory, the properties of the Hopf bifurcation at the critical value $\tau_{2 *}$ are determined. Let $t=s \tau$, $S(t)=u_{1}(s \tau), Q(t)=u_{2}(s \tau), I(t)=u_{3}(s \tau), R(t)=u_{4}(s \tau)$, and $\tau=\tau_{2 *}+\varrho$ where $\varrho \in R$. Throughout this section, we 
assume that $\tau_{1 *}<\tau_{2 *}$, where $\tau_{1 *} \in\left(0, \tau_{10}\right)$. Then, system (2) becomes the following form:

$$
\dot{u}(t)=L_{\varrho}\left(u_{t}\right)+F\left(\varrho, u_{t}\right),
$$

where $L_{\varrho}: C \longrightarrow R^{4}$ and $F: R \times C \longrightarrow R^{4}$ are defined, respectively, by

$$
\begin{aligned}
L_{\varrho} \phi= & \left(\tau_{2 *}+\varrho\right)\left(M_{1 \max } \phi(0)+M_{2 \max } \phi\left(-\frac{\tau_{1 *}}{\tau_{2 *}}\right)\right. \\
& \left.+M_{3 \max } \phi(-1)\right), \\
F(\varrho, \phi)= & \left(\begin{array}{c}
-\beta_{1} \phi_{1}(0) \phi_{3}(0) \\
-\beta_{2} \phi_{2}(0) \phi_{3}(0) \\
\beta_{1} \phi_{1}(0) \phi_{3}(0)+\beta_{2} \phi_{2}(0) \phi_{3}(0) \\
0
\end{array}\right),
\end{aligned}
$$

with

$$
\begin{aligned}
& M_{1 \max }=\left(\begin{array}{cccc}
L_{11} & 0 & L_{13} & 0 \\
0 & L_{22} & L_{23} & 0 \\
L_{31} & L_{32} & L_{33} & L_{34} \\
0 & 0 & L_{43} & L_{44}
\end{array}\right), \\
& M_{2 \max }=\left(\begin{array}{cccc}
0 & 0 & 0 & 0 \\
0 & 0 & M_{23} & 0 \\
0 & 0 & M_{33} & 0 \\
0 & 0 & 0 & 0
\end{array}\right), \\
& M_{3 \max }=\left(\begin{array}{llll}
0 & 0 & 0 & 0 \\
0 & 0 & 0 & N_{24} \\
0 & 0 & 0 & 0 \\
0 & 0 & 0 & N_{44}
\end{array}\right) .
\end{aligned}
$$

By the Riesz representation theorem in [35], there exists a function $\eta(\theta, \varrho)$ of bounded variation for $\theta \in[-1,0]$ such that

$$
L_{\varrho} \phi=\int_{-1}^{0} \mathrm{~d} \eta(\theta, \varrho) \phi(\theta) .
$$

In fact, one can choose

$$
\eta(\theta, \varrho)= \begin{cases}\left(\tau_{2 *}+\varrho\right)\left(M_{1 \max }+M_{2 \max }+M_{2 \max }\right), & \theta=0, \\ \left(\tau_{2 *}+\varrho\right)\left(M_{2 \max }+M_{3 \max }\right), & \theta \in\left[\frac{\tau_{1 *}}{\tau_{2 *}}, 0\right), \\ \left(\tau_{2 *}+\varrho\right) M_{3 \max }, & \theta \in\left(-1,-\frac{\tau_{1 *}}{\tau_{2 *}}\right), \\ 0, & \theta=-1,\end{cases}
$$

where $\delta$ is defined by

$$
\delta(\theta)= \begin{cases}0, & \theta \neq 0, \\ 1, & \theta=0\end{cases}
$$

For $\phi \in C\left([-1,0], R^{4}\right)$, define

$$
\begin{aligned}
& A(\varrho) \phi= \begin{cases}\frac{\mathrm{d} \phi(\theta)}{\mathrm{d} \theta}, & -1 \leq \theta<0, \\
\int_{-1}^{0} \mathrm{~d} \eta(\theta, \varrho) \phi(\theta), & \theta=0,\end{cases} \\
& R(\varrho) \phi= \begin{cases}0, & -1 \leq \theta<0, \\
F(\varrho, \phi), & \theta=0 .\end{cases}
\end{aligned}
$$

Then, system (48) is equivalent to

$$
\dot{u}(t)=A(\varrho) u_{t}+R(\varrho) u_{t},
$$

where $u_{t}=u(t+\theta)$.

The adjoint operator $A^{*}(\varrho)$ of $A(\varrho)$ is defined by

$$
A^{*}(\varphi)= \begin{cases}\frac{\mathrm{d} \phi(s)}{\mathrm{d} s}, & 0<s \leq 1, \\ \int_{-1}^{0} \mathrm{~d} \eta^{T}(s, 0) \phi(-s), & s=0 .\end{cases}
$$

For $\phi \in C\left([-1,0], R^{4}\right)$ and $\varphi \in C^{1}\left([0,1],\left(R^{4}\right)^{*}\right)$, define

$$
\begin{aligned}
\langle\varphi(s), \phi(\theta)\rangle= & \bar{\varphi}(0) \phi(0)-\int_{\theta=-1}^{0} \int_{\xi=0}^{\theta} \bar{\varphi}(\xi-\theta) \mathrm{d} \eta \\
& \cdot(\theta) \phi(\xi) \mathrm{d} \xi,
\end{aligned}
$$

where $\eta(\theta)=\eta(\theta, 0)$.

From Section 2, it can be seen that $\pm i \tau_{2 *} \omega_{2 *}$ are the eigenvalues of $A(0)$, so $\pm i \tau_{2 *} \omega_{2 *}$ are also the eigenvalues of $A^{*}(0)$. Suppose that $q(\theta)=\left(1, q_{2}, q_{3}, q_{4}\right)^{T} e^{i \tau_{2 *} \omega_{2 *} \theta}$ and $q^{*}(s)=D\left(1, q_{2}^{*}, q_{3}^{*}, q_{4}^{*}\right)^{T} e^{i \tau_{2 *} \omega_{2 *} s}$ be the eigenvectors for $A(0)$ and $A^{*}(0)$ corresponding to $+i \tau_{2 *} \omega_{2 *}$ and $-i \tau_{2 *} \omega_{2 *}$, respectively. Then, one can obtain

$$
\begin{aligned}
& q_{2}=\frac{L_{33}\left(i \omega_{2 *}-L_{11}\right)}{L_{13}\left(i \omega_{2 *}-L_{22}-M_{23} e^{-i \tau_{1 *} \omega_{2 *}}-N_{24} e^{-i \tau_{2 *} \omega_{2 *}}\right)}, \\
& q_{3}=\frac{i \omega_{2 *}-L_{11}}{L_{13}},
\end{aligned}
$$

$$
\begin{aligned}
& q_{4}=\frac{L_{43}\left(i \omega_{2 *}-L_{11}\right)}{L_{13}\left(i \omega_{2 *}-L_{44}-N_{24} e^{-i \tau_{2 *} \omega_{2 *}}\right)}, \\
& q_{2}^{*}=\frac{L_{11}\left(L_{32}+M_{33} e^{i \tau_{1 *} \omega_{2 *}}\right)}{\left(i \omega_{2 *}+L_{31}\right)\left(i \omega_{2 *}+L_{22}+M_{23} e^{i \tau_{1 *} \omega_{2 *}}\right)}, \\
& q_{3}^{*}=-\frac{L_{11}}{i \omega_{2 *}+L_{31}},
\end{aligned}
$$

$$
q_{4}^{*}=-\frac{N_{24} e^{i \tau_{2 *} \omega_{2 *}} q_{2}^{*}+L_{34} q_{3}^{*}}{i \omega_{2 *}+L_{44}+N_{44} e^{i \tau_{2 *} \omega_{2 *}}} .
$$


In order to guarantee $\left\langle q^{*}(s), q(\theta)\right\rangle=1$, the value of $D$ needs to be determined. In view of equation (57), we have

$$
\begin{aligned}
\bar{D}= & {\left[1+\sum_{i=1}^{4} q_{i}^{*} \bar{q}_{i}+\tau_{1 *} e^{i \tau_{1 *} \omega_{2 *}} \bar{q}_{2}\left(M_{23} q_{2}^{*}+M_{33} q_{3}^{*}\right)\right.} \\
& \left.+\tau_{2 *} e^{i \tau_{2 *} \omega_{2 *}} \bar{q}_{4}\left(N_{24} q_{2}^{*}+N_{44} Q_{4}^{*}\right)\right]^{-1} .
\end{aligned}
$$

In what follows, based on the algorithm in [36] and the similar computation process as that in [37-40], we can obtain the expressions of $g_{20}, g_{11}, g_{02}$, and $g_{21}$ as follows:

$$
\begin{aligned}
g_{20}= & 2 \bar{D} \tau_{2 *}\left(\bar{q}_{3}^{*}-1\right) \beta_{1} q_{3}+\left(\bar{q}_{3}^{*}-\bar{q}_{2}^{*}\right) \beta_{2} q_{2} q_{3}, \\
g_{11}= & \bar{D} \tau_{2 *}\left(\bar{q}_{3}^{*}-1\right) \beta_{1}\left(q_{3}+\bar{q}_{3}\right)+\left(\bar{q}_{3}^{*}-\bar{q}_{2}^{*}\right) \beta_{2}\left(q_{2} \bar{q}_{3}+\bar{q}_{2} q_{3}\right) \\
g_{02}= & 2 \bar{D} \tau_{2 *}\left(\bar{q}_{3}^{*}-1\right) \beta_{1} \bar{q}_{3}+\left(\bar{q}_{3}^{*}-\bar{q}_{2}^{*}\right) \beta_{2} \bar{q}_{2} \bar{q}_{3}, \\
g_{21}= & 2 \bar{D} \tau_{2 *}\left(\bar{q}_{3}^{*}-1\right) \beta_{1}\left(W_{11}^{(1)}(0) q_{3}+\frac{1}{2} W_{20}^{(1)}(0) \bar{q}_{3}+W_{11}^{(3)}(0)+\frac{1}{2} W_{20}^{(3)}(0)\right)+\left(\bar{q}_{3}^{*}-\bar{q}_{2}^{*}\right) \beta_{2} \\
& \cdot\left(W_{11}^{(2)}(0) q_{3}+\frac{1}{2} W_{20}^{(2)}(0) \bar{q}_{3}+W_{11}^{(3)}(0) q_{2}+\frac{1}{2} W_{20}^{(3)}(0) \bar{q}_{2}\right)
\end{aligned}
$$

with

$$
\begin{aligned}
& W_{20}(\theta)=\frac{i g_{20} q(0)}{\tau_{2 *} \omega_{2 *}} e^{i \tau_{2 *} \omega_{2 *} \theta}+\frac{i \bar{g}_{02} \bar{q}(0)}{3 \tau_{2 *} \omega_{2 *}} e^{-i \tau_{2 *} \omega_{2 *} \theta}+E_{1} e^{2 i \tau_{2 *} \omega_{2 *} \theta}, \\
& W_{11}(\theta)=-\frac{i g_{11} q(0)}{\tau_{2 *} \omega_{2 *}} e^{i \tau_{2 *} \omega_{2 *} \theta}+\frac{i \bar{g}_{11} \bar{q}(0)}{\tau_{2 *} \omega_{2 *}} e^{-i \tau_{2 *} \omega_{2 *} \theta}+E_{2} .
\end{aligned}
$$

$$
\begin{aligned}
& E_{1}=2\left(\begin{array}{cccc}
L_{11}^{*} & 0 & -L_{13} & 0 \\
0 & L_{22}^{*} & L_{23}^{*} & -N_{24} e^{-2 i \tau_{2 *} \omega_{2 *}} \\
L_{31} & -L_{32} & L_{33}^{*} & L_{34} \\
0 & 0 & -L_{43} & L_{44}^{*}
\end{array}\right)^{-1} \times\left(\begin{array}{c}
-\beta_{1} q_{3} \\
-\beta_{2} q_{2} q_{3} \\
\beta_{1} q_{3}+\beta_{2} q_{2} q_{3} \\
0
\end{array}\right), \\
& E_{2}=2\left(\begin{array}{cccc}
L_{11} & 0 & L_{13} & 0 \\
0 & L_{22} & L_{23}+M_{23} & N_{24} \\
L_{31} & L_{32} & L_{33}+M_{33} & L_{34} \\
0 & 0 & L_{43} & L_{44}+N_{44}
\end{array}\right)^{-1} \times\left(\begin{array}{c}
-\beta_{1}\left(q_{3}+\bar{q}_{3}\right) \\
-\beta_{2}\left(q_{2} \bar{q}_{3}+\bar{q}_{2} q_{3}\right) \\
\beta_{1}\left(q_{3}+\bar{q}_{3}\right)+\beta_{2}\left(q_{2} \bar{q}_{3}+\bar{q}_{2} q_{3}\right) \\
0
\end{array}\right),
\end{aligned}
$$

where

$$
\begin{aligned}
& L_{11}^{*}=2 i \omega_{2 *}-L_{11}, \\
& L_{22}^{*}=2 i \omega_{2 *}-L_{22}, \\
& L_{23}^{*}=-L_{23}-M_{23} e^{-2 i \tau_{1 *} \omega_{2 *},} \\
& L_{33}^{*}=2 i \omega_{2 *}-L_{33}-M_{33} e^{-2 i \tau_{1 *} \omega_{2 *}}, \\
& L_{44}^{*}=2 i \omega_{0}-L_{44}-N_{44} e^{-2 i \tau_{2 *} \omega_{2 *}},
\end{aligned}
$$

$$
\begin{aligned}
& \mu_{2}=-\frac{\operatorname{Re}\left\{C_{1}(0)\right\}}{\left\{\lambda^{\prime}\left(\tau_{2 *}\right)\right\}}, \\
& \beta_{2}=2 \operatorname{Re}\left\{C_{1}(0)\right\}, \\
& T_{2}=\frac{\operatorname{Im}\left\{C_{1}(0)\right\}+\mu_{2} \operatorname{Im}\left\{\lambda^{\prime}\left(\tau_{2 *}\right)\right\}}{\tau_{2 *} \omega_{2 *}} .
\end{aligned}
$$

Thus, we have 


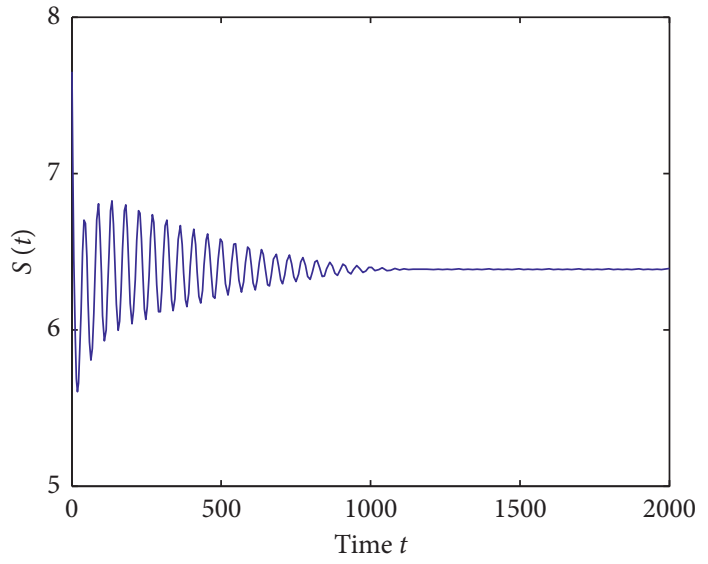

(a)

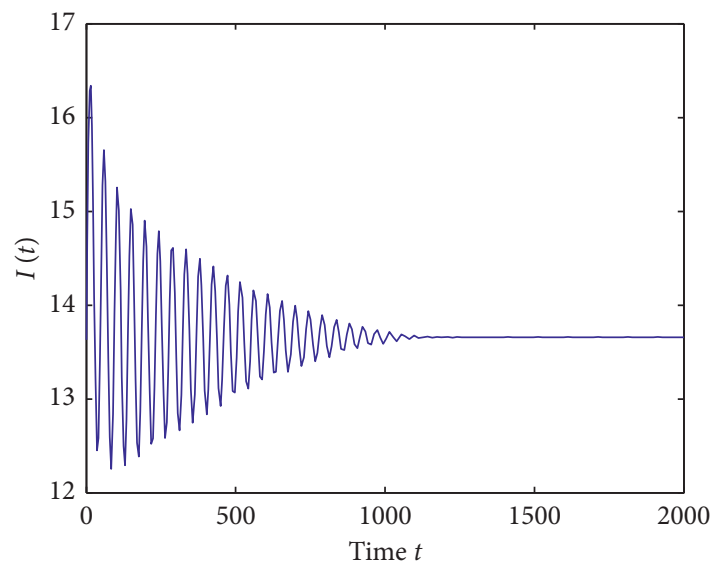

(c)

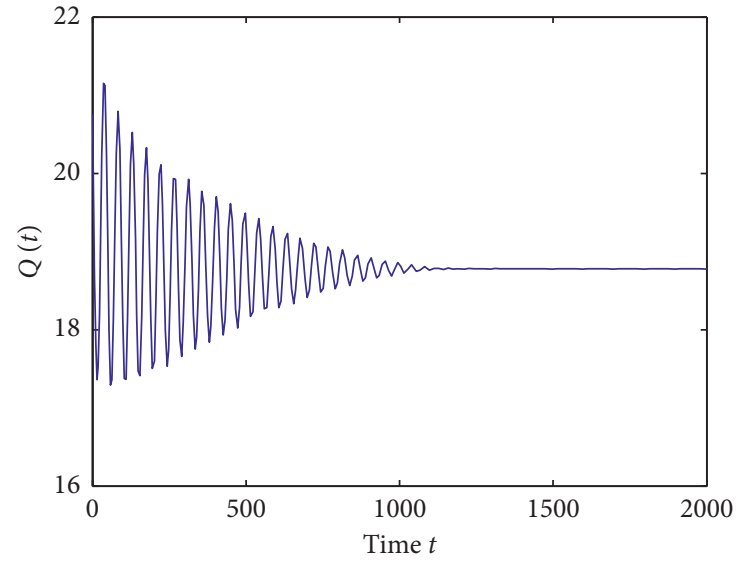

(b)

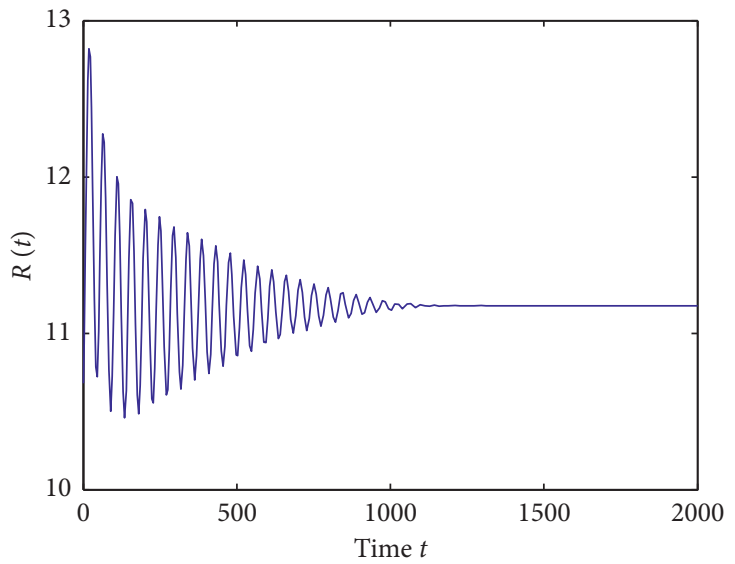

(d)

FIgURE 2: Waveform plots of system (65) with $\tau_{1}=15.2608<\tau_{10}$.

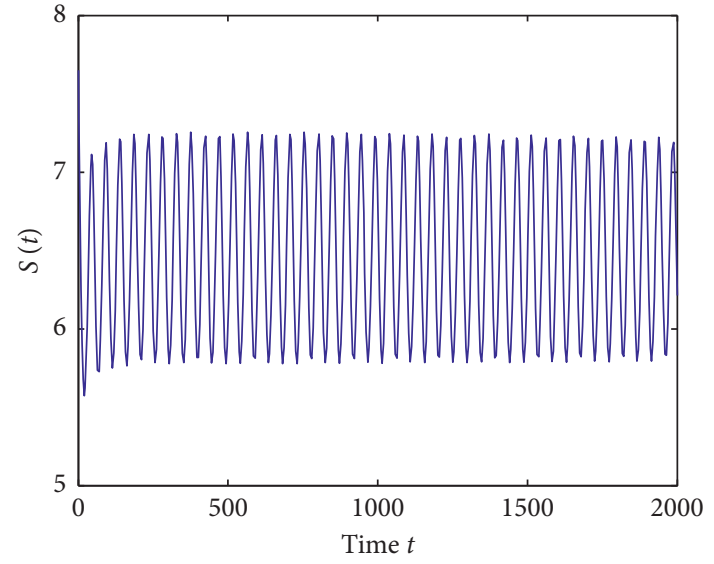

(a)

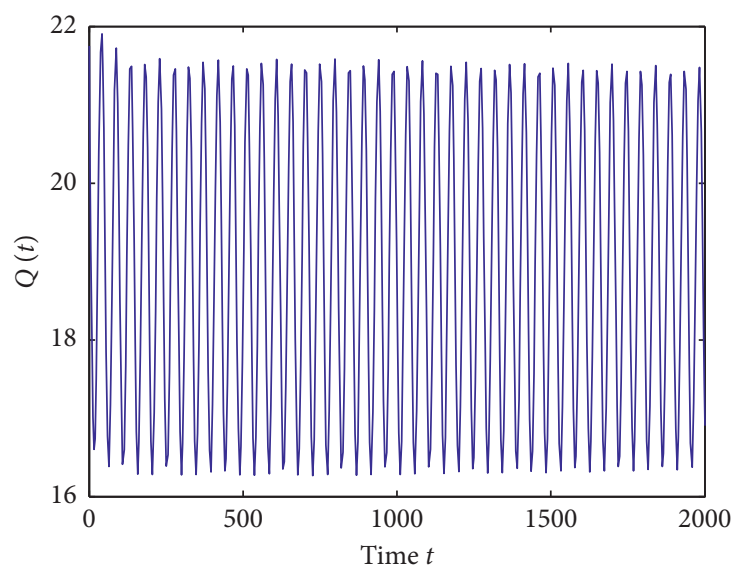

(b)

FIgUre 3: Continued. 


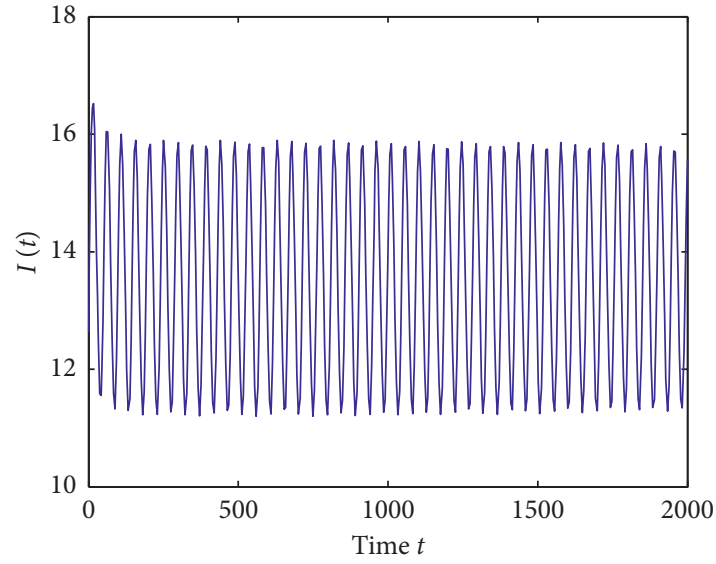

(c)

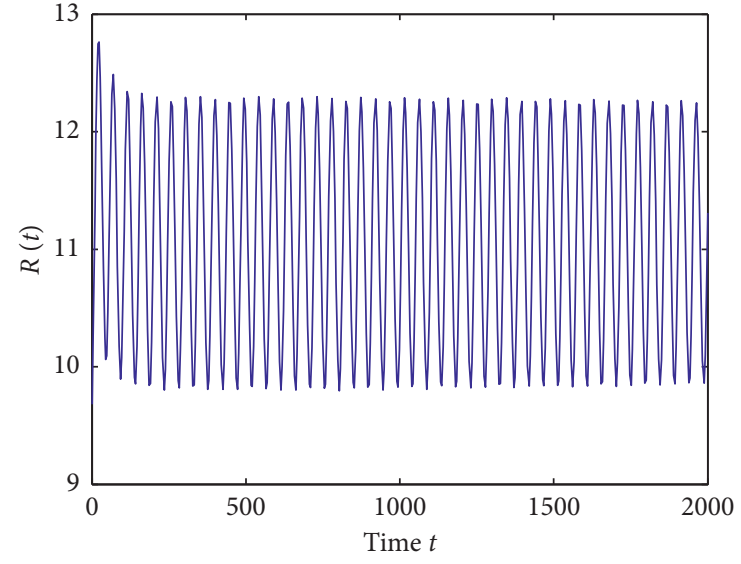

(d)

FIgURE 3: Waveform plots of system (65) with $\tau_{1}=16.3642>\tau_{10}$.

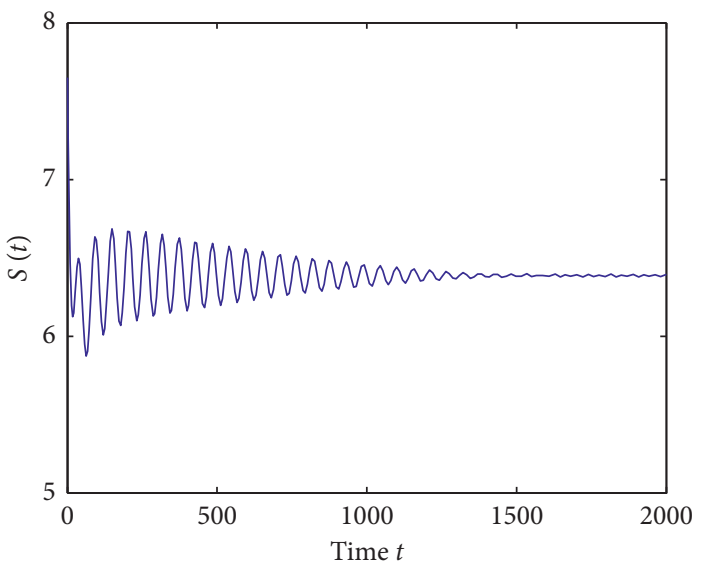

(a)

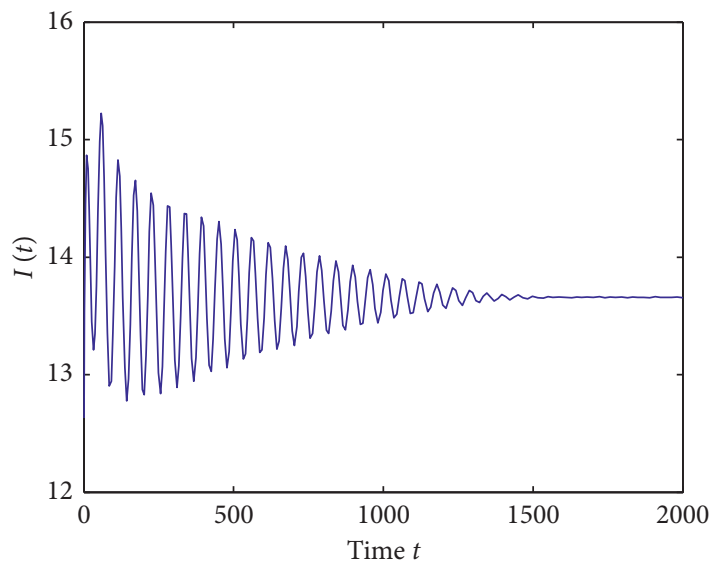

(c)

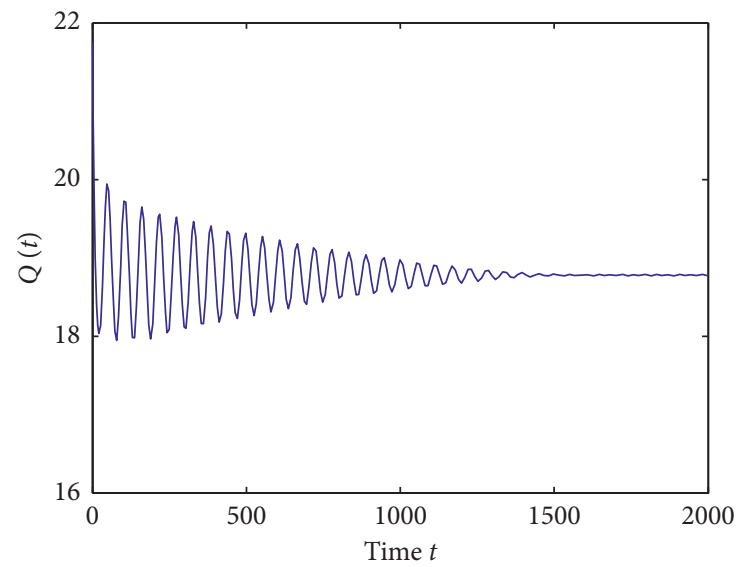

(b)

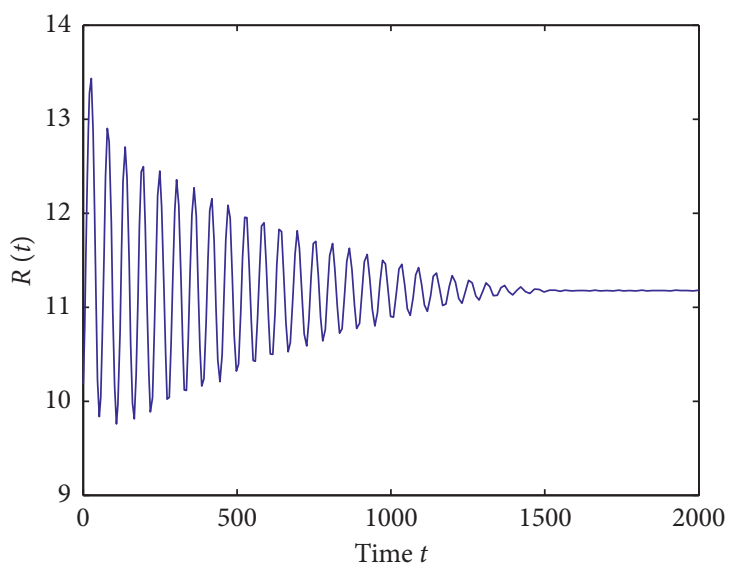

(d)

FIGURE 4: Waveform plots of system (65) with $\tau_{2}=20.3665<\tau_{20}$.

In conclusion, we can obtain the following results based on the fundamental results about Hopf bifurcation in the literature [32].
Theorem 4. For system (2), if $\mu_{2}>0\left(\mu_{2}<0\right)$, then the Hopf bifurcation is supercritical (subcritical); if $\beta_{2}<0\left(\beta_{2}>0\right)$, then the bifurcating periodic solutions are stable (unstable); if 


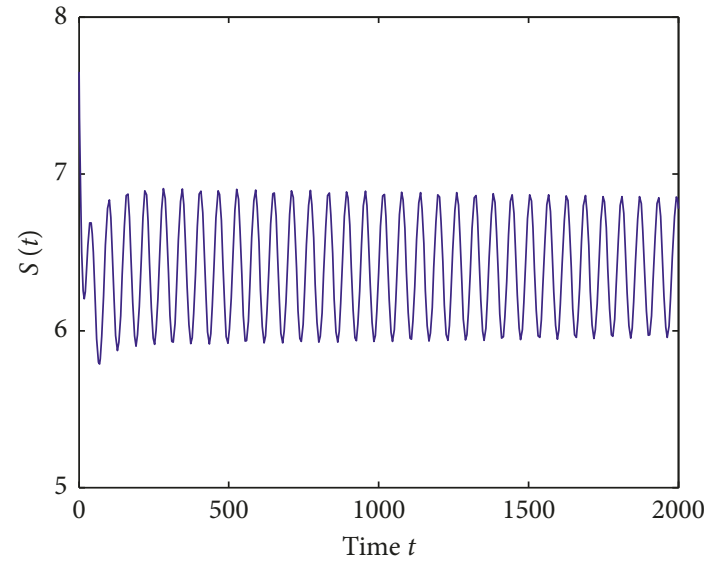

(a)



(c)

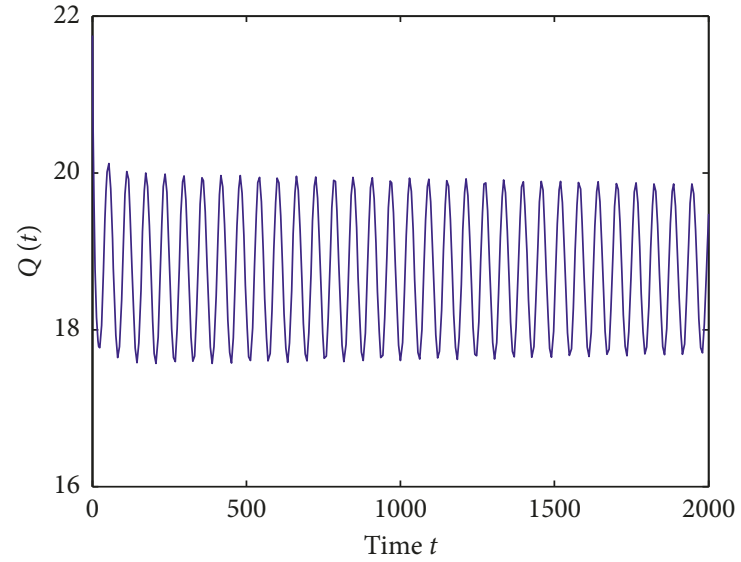

(b)

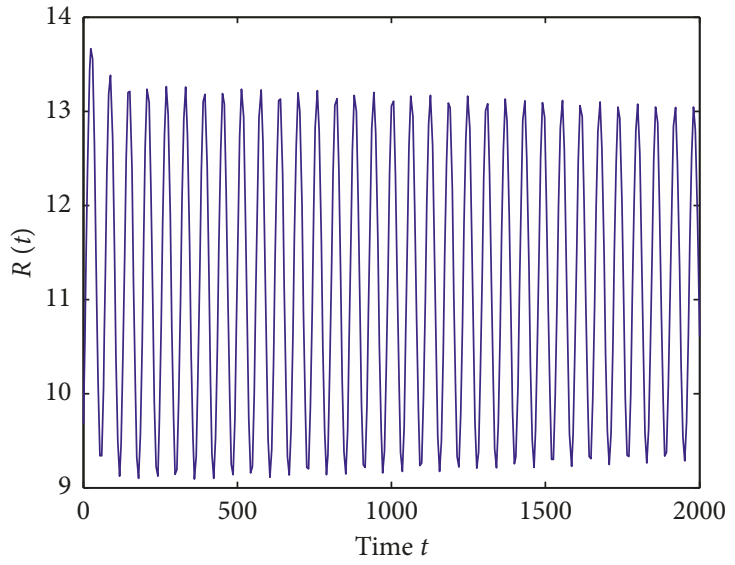

(d)

FIGURE 5: Waveform plots of system (65) with $\tau_{2}=23.9608>\tau_{20}$.

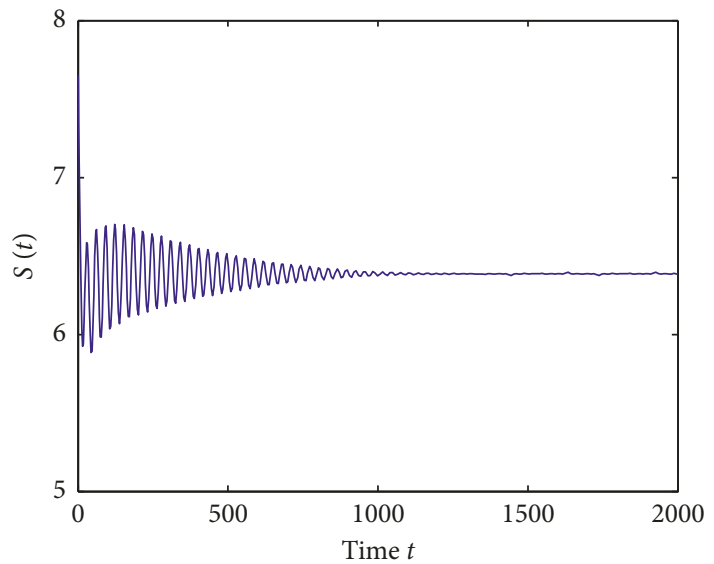

(a)

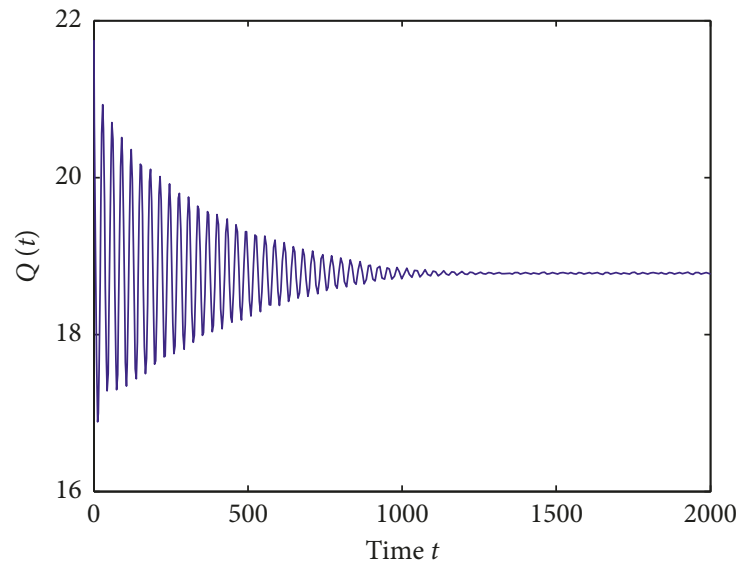

(b)

Figure 6: Continued. 


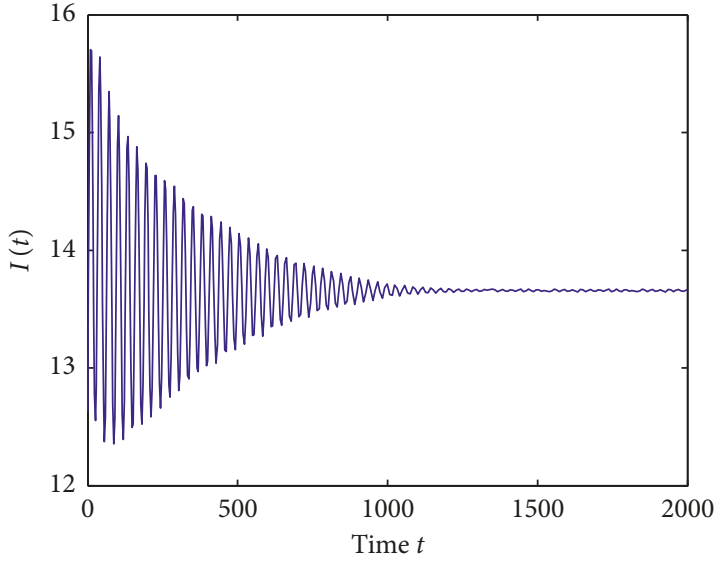

(c)

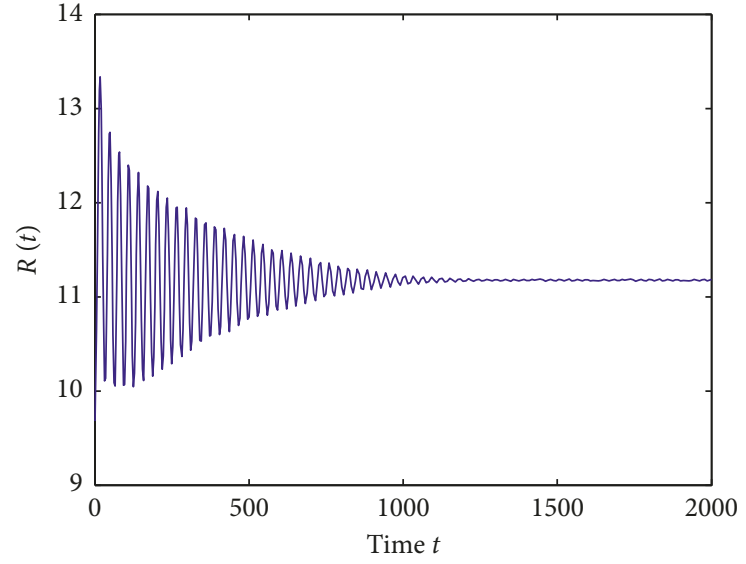

(d)

FIGURE 6: Waveform plots of system (65) with $\tau=8.4565<\tau_{0}$.

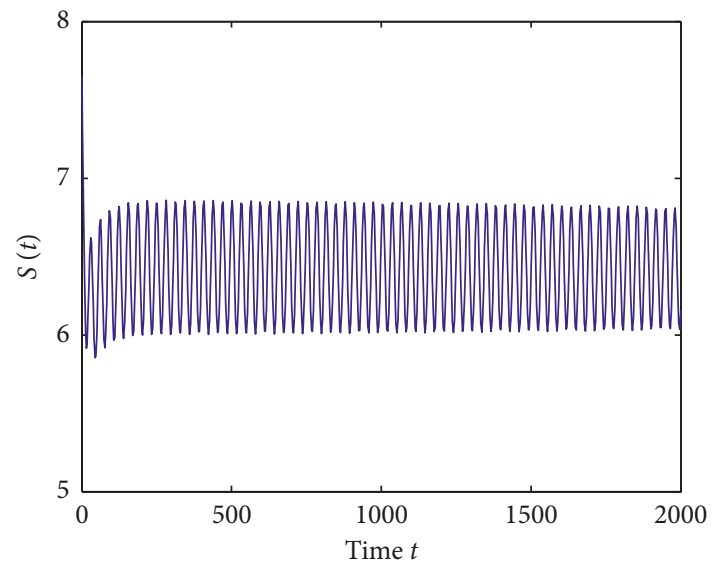

(a)

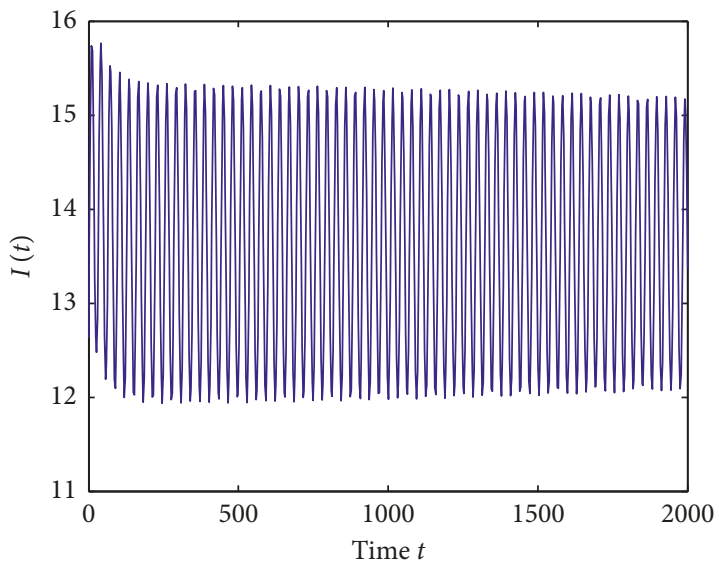

(c)

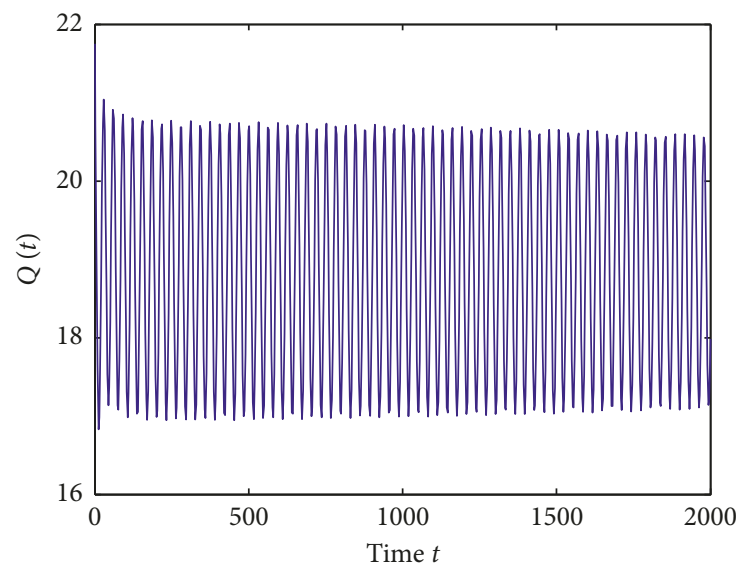

(b)

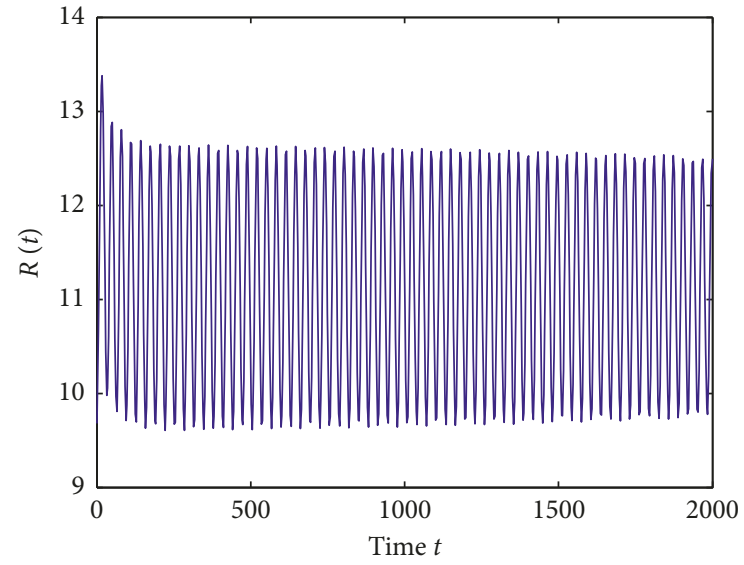

(d)

FIGURE 7: Waveform plots of system (65) with $\tau=8.7807>\tau_{0}$. 


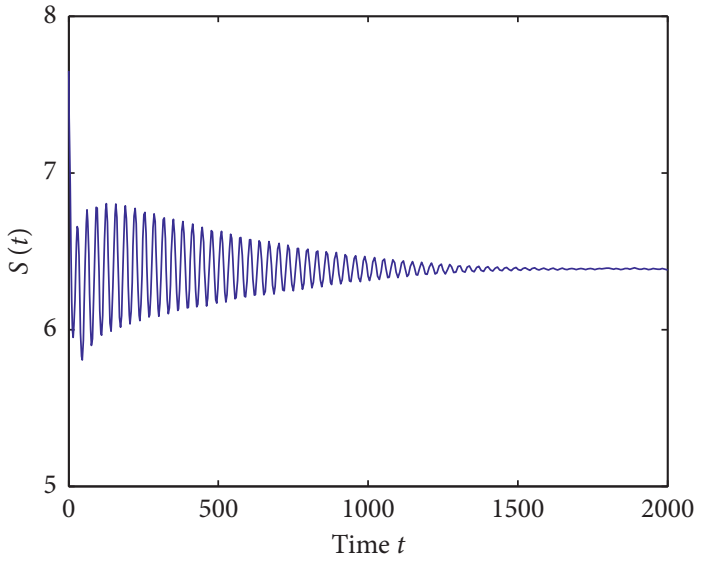

(a)

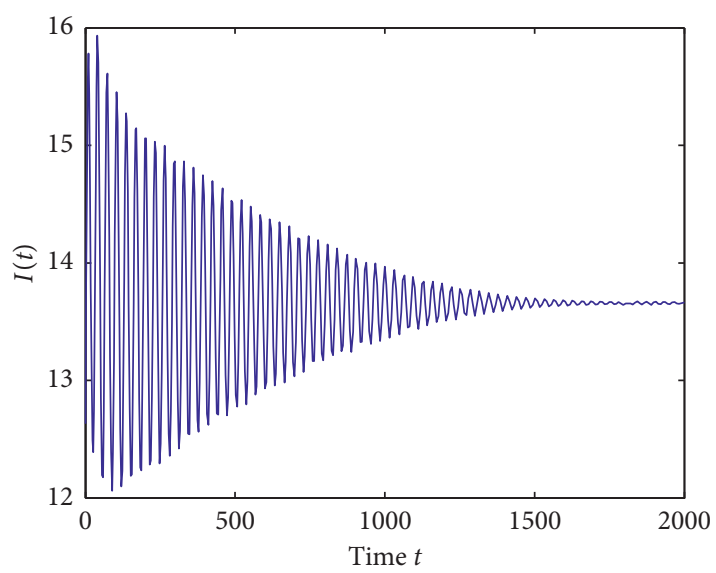

(c)

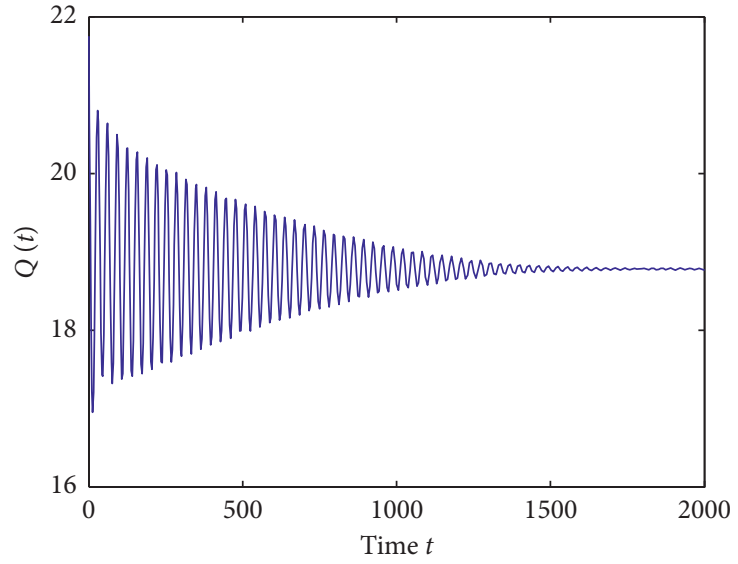

(b)

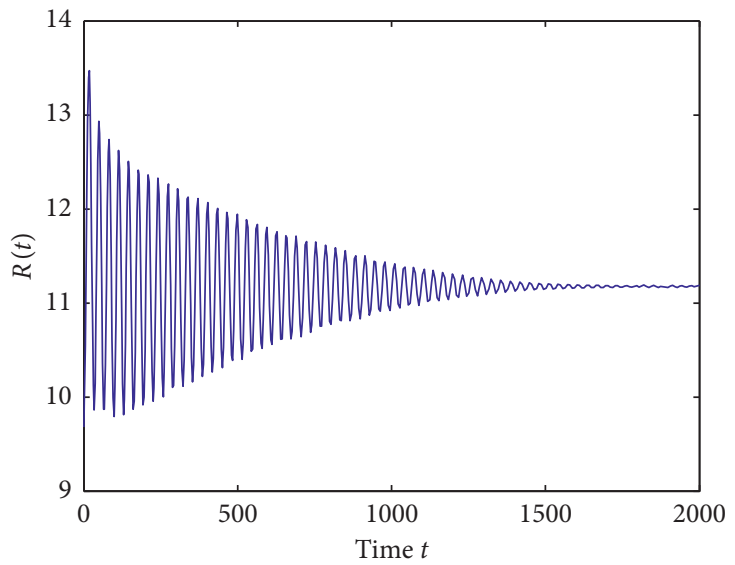

(d)

FIgURE 8: Waveform plots of system (65) with $\tau_{2}=10.2508<\tau_{2 *}$ and $\tau_{1}=7.75 \in\left(0, \tau_{10}\right)$.

$T_{2}>0\left(T_{2}<0\right)$, then the period of the bifurcating periodic solutions increases (decreases).

\section{Numerical Simulation}

In this section, numerical simulations are employed to confirm the efficiency of the theoretical analysis in the present paper. Considering the biological significance of the parameters in system (2), we refer to the range of the parameter values provided in the literature [26]. By extracting some values from the literature [26] and considering the biological significance of the parameters and conditions for the occurrence of Hopf bifurcation, we choose $\Lambda=1, \quad \beta_{1}=0.01, \quad \mu=0.02, \varepsilon=0.1, \quad \delta=0.095$, $\beta_{2}=0.008, \sigma=0.0011$, and $\gamma=0.095$. Then, system (2) turns into

$$
\left\{\begin{array}{l}
\frac{\mathrm{d} S(t)}{\mathrm{d} t}=1-0.01 S(t) I(t)-0.02 S(t), \\
\frac{\mathrm{d} Q(t)}{\mathrm{d} t}=0.1 I\left(t-\tau_{1}\right)+0.095 R\left(t-\tau_{2}\right) \\
-0.02 Q(t)-0.008 Q(t) I(t), \\
\frac{\mathrm{d} I(t)}{\mathrm{d} t}=0.01 S(t) I(t)+0.008 Q(t) I(t)+0.0011 R(t) \\
-0.115 I(t)-0.1 I\left(t-\tau_{1}\right), \\
\frac{\mathrm{d} R(t)}{\mathrm{d} t}=0.095 I(t)-0.0211 R(t)-0.095 R\left(t-\tau_{2}\right) .
\end{array}\right.
$$




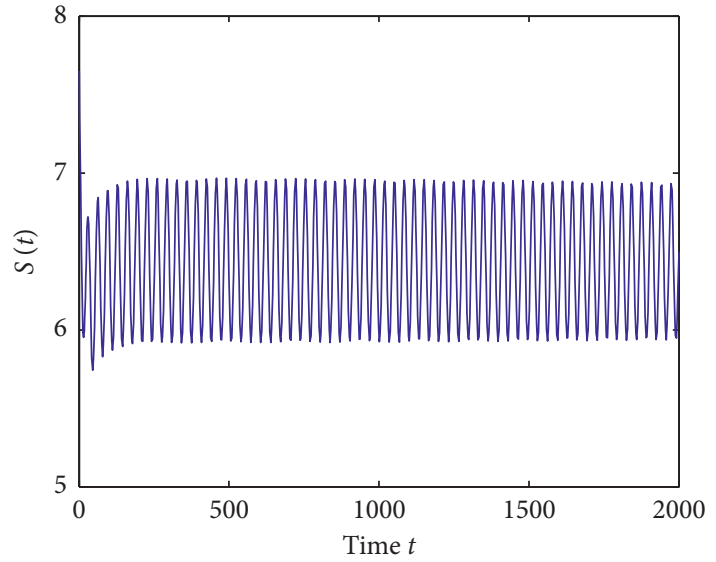

(a)

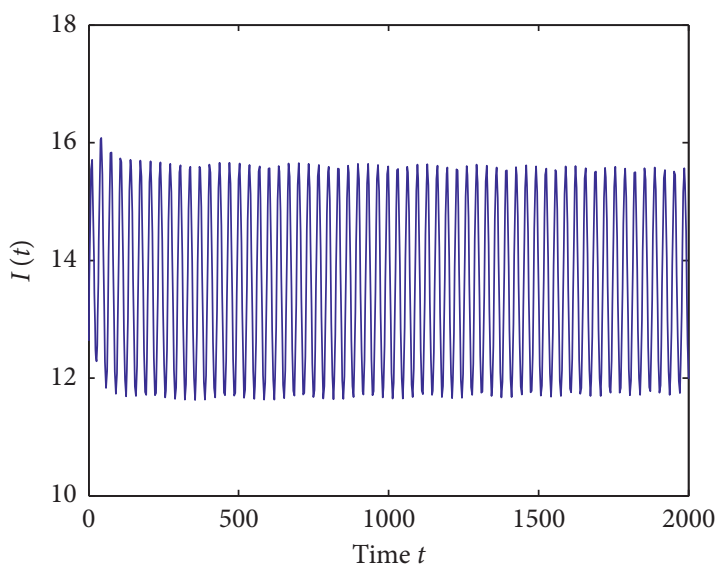

(c)

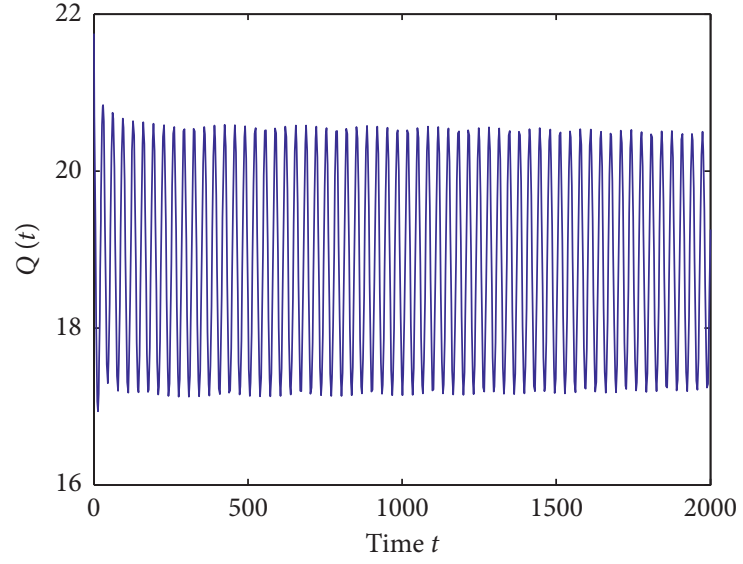

(b)

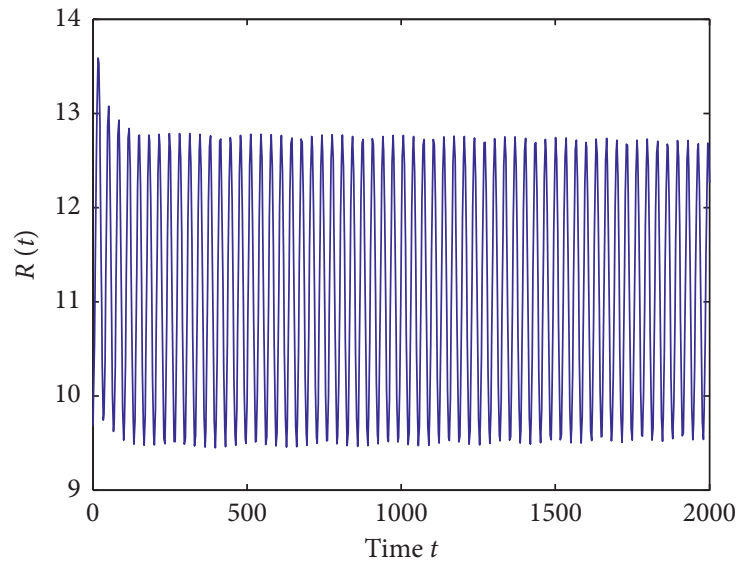

(d)

Figure 9: Waveform plots of system (65) with $\tau=11.3691>\tau_{2 *}$ and $\tau_{1}=7.75 \in\left(0, \tau_{10}\right)$.

Then, one can obtain $R_{0}=2.3530>1$, and equation (4) becomes the following form:

$$
3.3776 e-007\left(I^{*}\right)^{2}-3.6335 e-006 I^{*}-1.3277 e-005=0,
$$

from which one gets the unique positive root $I^{*}=13.6396$. Further, we obtain that system (65) has a unique drug-addiction equilibrium $E^{*}(6.3940,18.7755,13.6396$, 11.1607). By delicate calculation, it is obtained that $\omega_{10}=$ 0.1872 and $\tau_{10}=16.0145 ; \omega_{20}=0.9243$ and $\tau_{20}=21.9566$; $\omega_{0}=2.9207$ and $\tau_{0}=8.6947 ; \quad \omega_{2 *}=1.4648$ and $\tau_{2 *}=10.7568$ when $\tau_{1 *}=7.75 \in\left(0, \tau_{10}\right)$.

By Theorem $1, E^{*}(6.3940,18.7755,13.6396,11.1607)$ is asymptotically stable for system (65) when $\tau_{1}=$ $15.2608<\tau_{10}$, which is depicted in Figure 2. $E^{*}(6.3940$, $18.7755,13.6396,11.1607)$ is unstable for system (65) and Hopf bifurcation occurs when $\tau_{1}=16.3642>\tau_{10}$, which are simulated in Figure 3. Similar simulations can be shown as in Figures 4 and 5 for Theorem 2, Figures 6 and 7 for Theorem 3, and Figures 8 and 9 for Theorem 4, respectively.

Whereafter, by some complex calculations, we obtain $C_{1}(0)=-13.0664+9.6207 i$ and $\lambda^{\prime}\left(\tau_{2 *}\right)=1.0081-0.6309 i$. By the results in equation (64), it can be derived that $\mu_{2}=12.9614>0, \beta_{2}=-26.1328<0$, and $T_{2}=-0.0916<0$. It follows from Theorem 4 that the Hopf bifurcation is supercritical since $\mu_{2}>0$, the bifurcating periodic solutions are stable since $\beta_{2}<0$, and the period of the periodic solutions decreases as $\tau_{2}$ increases since $T_{2}<0$.

\section{Conclusions}

In this paper, a delayed synthetic drug transmission model with relapse and treatment is investigated by incorporating two delays into the model proposed in the literature [26]. We consider not only the time delay due to the period that the drug abusers use to give up drugs through self-control but also the time delay due to the period used to give up drugs through successful treatment. Compared with the model in [26], the delayed synthetic drug transmission model in the present paper is more general because it usually needs a period for the drug abusers to give up drugs through either self-control or successful treatment.

It has been shown that, under certain conditions, the drug-addiction equilibrium is locally asymptotically stable when the value of the time delay is below the critical value. In this case, system (2) is in ideal stable state and the synthetic drug transmission can be controlled easily. However, once the value of the time delay is above the critical value, system 
(2) will lose its stability and undergo a Hopf bifurcation at the corresponding critical value of the time delay, which is not welcomed in reality. The occurrence of Hopf bifurcation means that the existence of populations in system (2) changes from the drug-addiction equilibrium to a limit cycle, and in this case, the synthetic drug transmission is out of control. Therefore, it is vital to take some necessary measures to postpone and eliminate the occurrence of the Hopf bifurcation for system (2).

Specially, the direction of the Hopf bifurcation and stability of the bifurcating periodic solutions are determined by employing the center manifold theorem and normal form theory. In addition, according to the numerical simulations, it is easily observed that the time delay due to the period that the drug abusers use to give up drugs through self-control is marked because the critical value of $\tau_{1}$ is much smaller when we only consider it. From this point of view, it is strongly recommended that drug abusers should have strong will in the process of giving up drugs.

\section{Data Availability}

The data used to support the findings of this study are available from the corresponding author upon request.

\section{Conflicts of Interest}

The authors declare that there are no conflicts of interest regarding the publication of this paper.

\section{Acknowledgments}

This study was supported by the Project of Support Program for Excellent Youth Talent in Colleges and Universities of Anhui Province (no. gxyqZD2018044), Natural Science Foundation of Anhui Province (no. 1708085MA17), and Natural Science Foundation of the Higher Education Institutions of Anhui Province (nos. KJ2019A0655, KJ2019A0656, and KJ2019A0662).

\section{References}

[1] S. T. Liu, L. Zhang, X. B. Zhang, and A. B. Li, "Dynamics of a stochastic heroin epidemic model with bilinear incidence and varying population size," International Journal of Biomathematics, vol. 12, no. 1, p. 21, 2019.

[2] Y. C. Wei, Q. G. Yang, and G. J. Li, "Dynamics of the stochastically perturbed heroin epidemic model under nondegenerate noises," Physica A: Statistical Mechanics and Its Applications, vol. 526, Article ID 120914, p. 10, 2019.

[3] X. Liu and J. Wang, "Epidemic dynamics on a delayed multigroup heroin epidemic model with nonlinear incidence rate," Journal of Nonlinear Sciences and Applications, vol. 09, no. 05, pp. 2149-2160, 2016.

[4] 2019, http://www.hqrw.com.cn/2019/0627/87860.shtml.

[5] D. R. Mackintosh and G. T. Stewart, "A mathematical model of a heroin epidemic: implications for control policies," Journal of Epidemiology \& Community Health, vol. 33, no. 4, pp. 299-304, 1979.

[6] S. Saha and G. P. Samanta, "Dynamics of an epidemic model with impact of toxins," Physica A: Statistical Mechanics and Its Applications, vol. 527, Article ID 121152, 2019.
[7] E. White and C. Comiskey, "Heroin epidemics, treatment and ODE modelling," Mathematical Biosciences, vol. 208, no. 1, pp. 312-324, 2007.

[8] G. Mulone and B. Straughan, "A note on heroin epidemics," Mathematical Biosciences, vol. 218, no. 2, pp. 138-141, 2009.

[9] X. Wang, J. Yang, and X. Li, "Dynamics of a heroin epidemic model with very population," Applied Mathematics, vol. 02, no. 06, pp. 732-738, 2011.

[10] J. Liu and T. Zhang, "Global behaviour of a heroin epidemic model with distributed delays," Applied Mathematics Letters, vol. 24, no. 10, pp. 1685-1692, 2011.

[11] G. Huang and A. Liu, "A note on global stability for a heroin epidemic model with distributed delay," Applied Mathematics Letters, vol. 26, no. 7, pp. 687-691, 2013.

[12] I. M. Wangari and L. Stone, "Analysis of a heroin epidemic model with saturated treatment function," Journal of Applied Mathematics, vol. 2017, Article ID 1953036, 21 pages, 2017.

[13] J. Y. Yang, X. X. Li, and F. Q. Zhang, "Global dynamics of a heroin epidemic model with age structure and nonlinear incidence," International Journal of Biomathematics, vol. 9, no. 3, Article ID 1650033, p. 20, 2016.

[14] M. Ma, S. Liu, and J. Li, "Bifurcation of a heroin model with nonlinear incidence rate," Nonlinear Dynamics, vol. 88, no. 1 , pp. 555-565, 2017.

[15] S. Djilali, T. M. Touaoula, and S. E.-H. Miri, "A heroin epidemic model: very general non linear incidence, treat-age, and global stability," Acta Applicandae Mathematicae, vol. 152, no. 1, pp. 171-194, 2017.

[16] B. Fang, X.-Z. Li, M. Martcheva, and L.-M. Cai, "Global asymptotic properties of a heroin epidemic model with treatage," Applied Mathematics and Computation, vol. 263, pp. 315-331, 2015.

[17] B. Fang, X. Li, M. Martcheva, and L. Cai, "Global stability for a heroin model with age-dependent susceptibility," Journal of Systems Science and Complexity, vol. 28, no. 6, pp. 1243-1257, 2015.

[18] J. Wang, J. Wang, and T. Kuniya, "Analysis of an agestructured multi-group heroin epidemic model," Applied Mathematics and Computation, vol. 347, pp. 78-100, 2019.

[19] S. Liu, L. Zhang, and Y. Xing, "Dynamics of a stochastic heroin epidemic model," Journal of Computational and Applied Mathematics, vol. 351, pp. 260-269, 2019.

[20] X. Abdurahman, L. Zhang, and Z. D. Teng, "Global dynamics of a discretized heroin epidemic model with time delay," Abstract and Applied Analysis, vol. 2014, Article ID 742385, 10 pages, 2014.

[21] G. P. Samanta, "Dynamic behaviour for a nonautonomous heroin epidemic model with time delay," Journal of Applied Mathematics and Computing, vol. 35, no. 1-2, pp. 161-178, 2011.

[22] G. P. Samanta and S. Sharma, "Dynamic behaviour for heroin epidemic models," in Heroin: Pharmacology, Effects and Abuse Prevention, M. B. Guthrie and B. M. Wooten, Eds., pp. 51-91, Nova Science Publishers Inc., New York, NY, USA, 2012.

[23] 2018, http://www.nncc626.com/2019-06/17/c_1210161797.htm.

[24] M. Ma, S. Liu, H. Xiang, and J. Li, "Dynamics of synthetic drugs transmission model with psychological addicts and general incidence rate," Physica A: Statistical Mechanics and Its Applications, vol. 491, pp. 641-649, 2018.

[25] S. Saha and G. P. Samanta, "Synthetic drugs transmission: stability analysis and optimal control," Letters in Biomathematics, vol. 6, 2019.

[26] P. Liu, L. Zhang, and Y. Xing, "Modelling and stability of a synthetic drugs transmission model with relapse and 
treatment," Journal of Applied Mathematics and Computing, vol. 60, no. 1-2, pp. 465-484, 2019.

[27] J. Ren, X. Yang, L.-X. Yang, Y. Xu, and F. Yang, "A delayed computer virus propagation model and its dynamics," Chaos, Solitons \& Fractals, vol. 45, no. 1, pp. 74-79, 2012.

[28] L. Wang, R. Xu, and G. Feng, "Modelling and analysis of an eco-epidemiological model with time delay and stage structure," Journal of Applied Mathematics and Computing, vol. 50, no. 1-2, pp. 175-197, 2016.

[29] L. Wen and X. Yang, "Global stability of a delayed SIRS model with temporary immunity," Chaos, Solitons \& Fractals, vol. 38, no. 1, pp. 221-226, 2008.

[30] X. G. Sun and J. J. Wei, "Stability and bifurcation analysis in a viral infection model with delays," Advances in Difference Equations, vol. 332, p. 22, 2015.

[31] N. Keshri and B. K. Mishra, "Two time-delay dynamic model on the transmission of malicious signals in wireless sensor network," Chaos, Solitons \& Fractals, vol. 68, pp. 151-158, 2014.

[32] Y. Z. Bai and Y. Y. Li, "Stability and Hopf bifurcation for a stage-structured predator-prey model incorporating refuge for prey and additional food for predator," Advances in Difference Equations, vol. 42, pp. 1-20, 2019.

[33] X. B. Zhang and H. L. Zhu, "Hopf bifurcation and chaos of a delayed finance system," Complexity, vol. 2019, Article ID 6715036, 18 pages, 2019.

[34] X.-Y. Meng, H.-F. Huo, X.-B. Zhang, and H. Xiang, "Stability and Hopf bifurcation in a three-species system with feedback delays," Nonlinear Dynamics, vol. 64, no. 4, pp. 349-364, 2011.

[35] J. J. Benedetto and W. Czaja, Riesz Representation Theorem, Birkhauser, Boston, MA, USA, 2009.

[36] B. D. Hassard, N. D. Kazarinoff, and Y. H. Wan, Theory and Applications of Hopf Bifurcation, Cambridge, Cambridge University Press, Cambridge, UK, 1981.

[37] X. Zhang, Z. Z. Zhang, and M. J. Wade, "Dynamical analysis of a competition and cooperation system with multiple delays," Boundary Value Problems, vol. 2018, no. 1, p. 111, 2018.

[38] C. Bianca, M. Ferrara, and L. Guerrini, "The Cai model with time delay: existence of periodic solutions and asymptotic analysis," Applied Mathematics \& Information Sciences, vol. 7, no. 1, pp. 21-72, 2013.

[39] C. J. Xu, "Delay-induced oscillations in a competitor-competitor-mutualist Lotka-Volterra model," Complexity, vol. 2017, Article ID 2578043, 12 pages, 2017.

[40] Z. Cheng and J. Cao, "Hybrid control of Hopf bifurcation in complex networks with delays," Neurocomputing, vol. 131, pp. 164-170, 2014. 


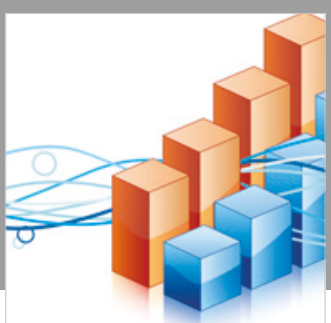

Advances in

Operations Research

\section{-n-m}
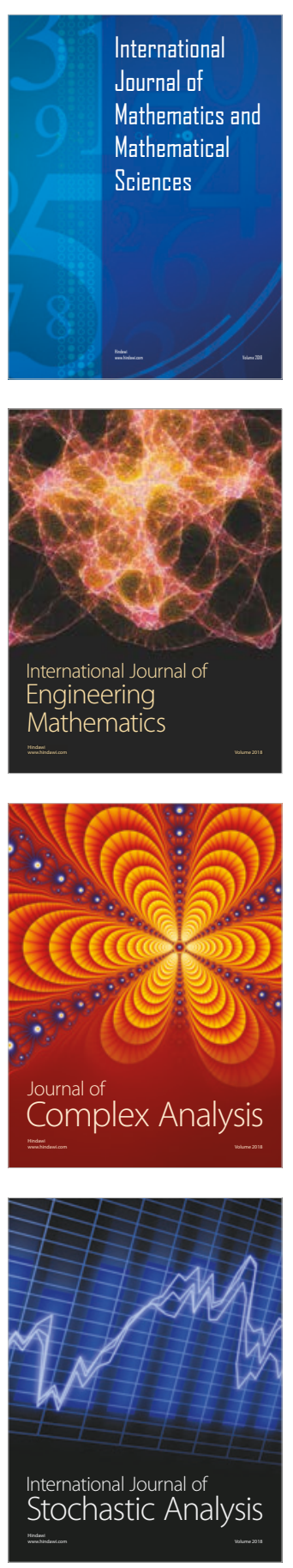


Journal of

Applied Mathematics
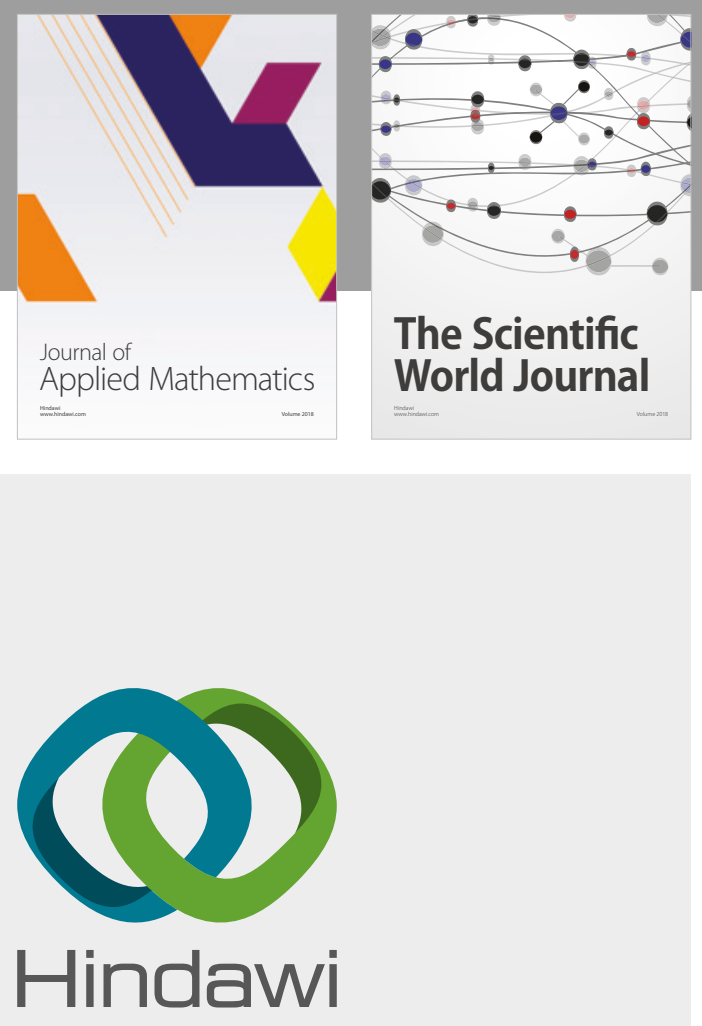

Submit your manuscripts at

www.hindawi.com

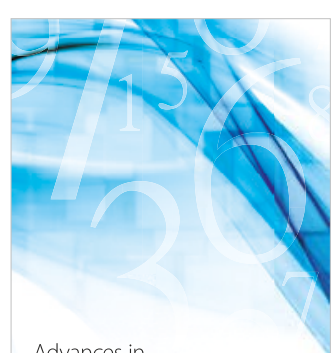

Advances in
Numerical Analysis
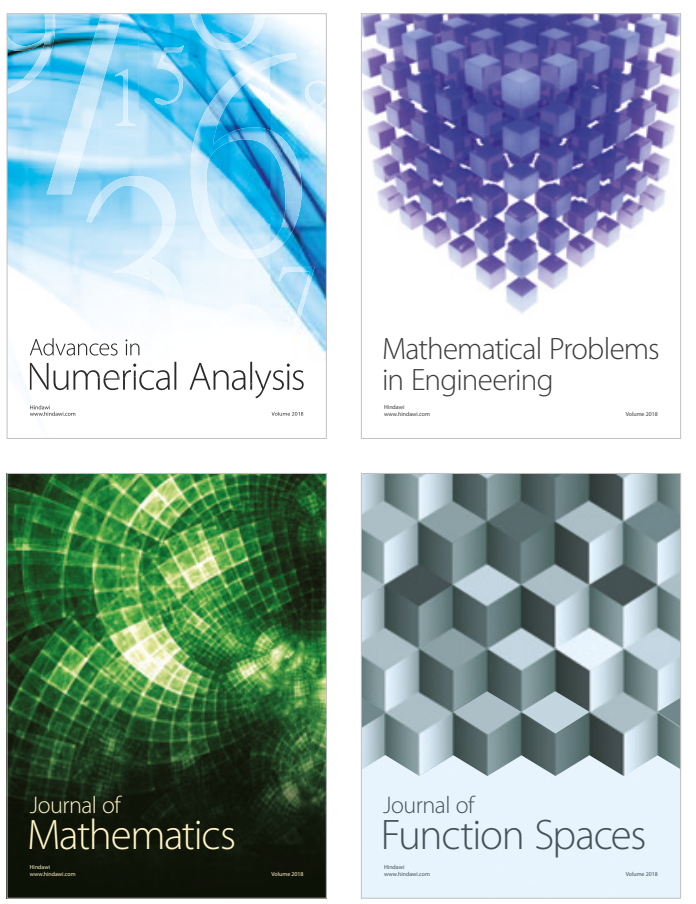

Mathematical Problems in Engineering

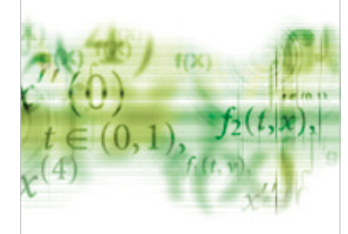

International Journal of

Differential Equations

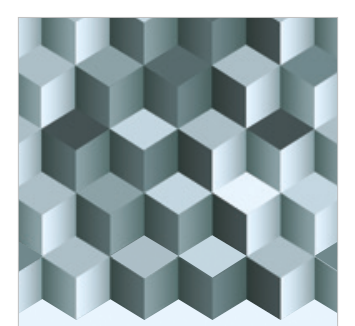

Journal of

Function Spaces
The Scientific

World Journal

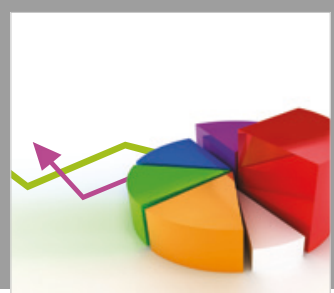

Journal of

Probability and Statistics
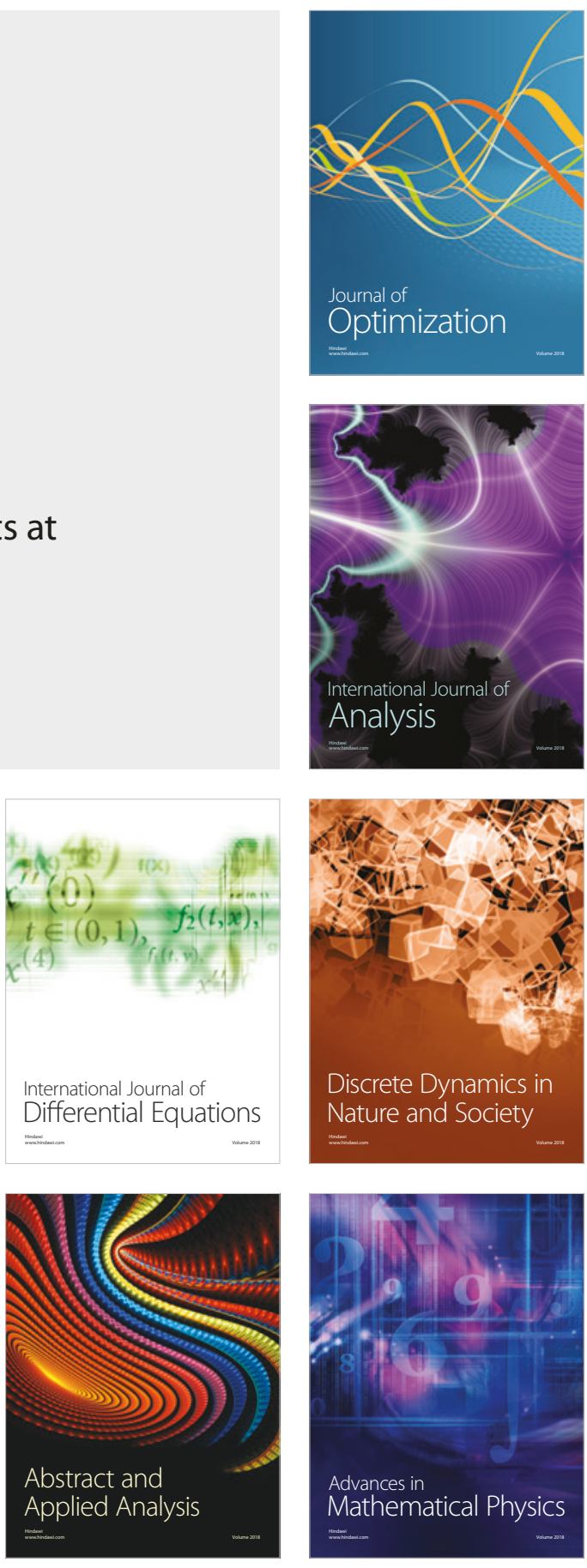